\title{
A Nitroalkene Benzoic Acid Derivative Targets Reactive Microglia and Prolongs Survival in an Inherited Model of ALS via NF-KB Inhibition
}

\author{
Sofía Ibarburu ${ }^{1}$ - Mariángeles Kovacs ${ }^{1}$ - Valentina Varela ${ }^{1}$ - Jorge Rodríguez-Duarte ${ }^{2}$ - Mariana Ingold ${ }^{2,3}$. \\ Paulina Invernizzi ${ }^{2}$. Williams Porcal ${ }^{2,3}$. Ana Paula Arévalo ${ }^{4}$. Karen Perelmuter ${ }^{5}$ - Mariela Bollati-Fogolín ${ }^{5}$. \\ Carlos Escande ${ }^{6}$. Gloria V. López ${ }^{2,3} \cdot$ Peter King ${ }^{7,8} \cdot$ Ying Si $^{7,8} \cdot$ Yuri Kwon ${ }^{7} \cdot$ Carlos Batthyány $^{2} \cdot$ Luis Barbeito $^{1}$. \\ Emiliano Trias ${ }^{1}$
}

Accepted: 12 October 2020 / Published online: 28 October 2020

(C) The American Society for Experimental NeuroTherapeutics, Inc. 2020

\begin{abstract}
Motor neuron degeneration and neuroinflammation are the most striking pathological features of amyotrophic lateral sclerosis (ALS). ALS currently has no cure and approved drugs have only a modest clinically therapeutic effect in patients. Drugs targeting different deleterious inflammatory pathways in ALS appear as promising therapeutic alternatives. Here, we have assessed the potential therapeutic effect of an electrophilic nitroalkene benzoic acid derivative, (E)-4-(2-nitrovinyl) benzoic acid (BANA), to slow down paralysis progression when administered after overt disease onset in SOD $1^{\mathrm{G} 93 \mathrm{~A}}$ rats. BANA exerted a significant inhibition of NF- $\mathrm{KB}$ activation in NF-KB reporter transgenic mice and microglial cell cultures. Systemic daily oral administration of BANA to $\mathrm{SOD} 1^{\mathrm{G} 93 \mathrm{~A}}$ rats after paralysis onset significantly decreased microgliosis and astrocytosis, and significantly reduced the number of NF-kB-p65-positive microglial nuclei surrounding spinal motor neurons. Numerous microglia bearing nuclear NF-KB-p65 were observed in the surrounding of motor neurons in autopsy spinal cords from ALS patients but not in controls, suggesting ALS-associated microglia could be targeted by BANA. In addition, BANA-treated SOD1 ${ }^{\mathrm{G} 93 \mathrm{~A}}$ rats after paralysis onset showed significantly ameliorated spinal motor neuron pathology as well as conserved neuromuscular junction innervation in the skeletal muscle, as compared to controls. Notably, BANA prolonged post-paralysis survival by $\sim 30 \%$, compared to vehicle-treated littermates. These data provide a rationale to therapeutically slow paralysis progression in ALS using small electrophilic compounds such as BANA, through a mechanism involving microglial NF- $\mathrm{kB}$ inhibition.
\end{abstract}

Key Words ALS $\cdot$ NF-kB-p65 $\cdot$ microglia $\cdot$ BANA.

\section{Introduction}

There is no current treatment to stop disease progression in amyotrophic lateral sclerosis (ALS), a paralytic disease

Luis Barbeito

barbeito@pasteur.edu.uy

Emiliano Trias

etrias@pasteur.edu.uy

1 Neurodegeneration Laboratory, Institut Pasteur de Montevideo, Mataojo, 2020 Montevideo, Uruguay

2 Laboratory of Vascular Biology and Drug Development, INDICYO Program, Institut Pasteur de Montevideo, Montevideo, Uruguay

3 Departmento de Química Orgánica, Facultad de Química, Universidad de la República, Montevideo, Uruguay characterized by progressive degeneration of motor neurons that control skeletal muscles. Survival after diagnosis average 3 to 5 years, and is largely determined by the rate of spread of motor neuron pathology along the neuroaxis [1]. ALS

Transgenic and Experimental Animal Unit, Institut Pasteur de Montevideo, Montevideo, Uruguay

5 Cell Biology Unit, Institut Pasteur de Montevideo, Montevideo, Uruguay

6 Laboratory of Metabolic Diseases and Aging, INDICyO Program, Institut Pasteur Montevideo, Montevideo, Uruguay

7 Department of Neurology, University of Alabama at Birmingham, Birmingham, AL 35294, USA

8 Birmingham Veterans Affairs Medical Center, Birmingham, AL 35294, USA 
etiology remains largely unknown and there is a poor understanding of the pathological mechanisms underlying disease onset and subsequent progressive spreading. Currently approved drugs for ALS such as riluzole and edaravone, have only modest, if any, therapeutic effects in most patients. Clinical trials have shown that riluzole extends survival by a few months [2], while edaravone improves the daily functioning in a restricted subset of ALS subjects [3]. Therefore, there is an unmet medical need to develop new treatments to slow the progression of the paralysis early after diagnosis, with the hope of turning this fatal disease into a chronic condition.

In the SOD $1^{\mathrm{G} 93 \mathrm{~A}}$ mutant rat model of ALS, the spread of paralysis is associated with marked glial cell activation [4] and the emergence of aberrant glial cells that actively proliferate around degenerating motor neurons [5-7]. Furthermore, aberrant glial cells display a marked neurotoxic potential on cultured motor neurons [5], which might further contribute to paralysis progression in ALS rats. Pharmacological downregulation of microglia and aberrant glial cells using tyrosine kinase inhibitors such as masitinib has shown to slow paralysis progression in $\mathrm{SOD} 1^{\mathrm{G} 93 \mathrm{~A}}$ rats, even when treatment starts up to 7 days after disease onset [8]. Masitinib has proved preliminary significant clinical efficacy in a subset of ALS patients [9]. Therefore, drugs targeting dysfunctional or reactive microglial cells that abnormally interact with motor neurons in ALS appear as a valid strategy to prevent neuronal loss and slow paralysis progression.

The transcription nuclear factor $k$ light-chain-enhancer of activated $\mathrm{B}$ cells $(\mathrm{NF}-\mathrm{KB})$ is widely distributed among various cell types in the CNS being involved in many physiological and pathological processes $[10,11]$. NF-KB is constitutively expressed as a dimer usually formed by subunits $\mathrm{p} 50$ and $\mathrm{p} 65$, which dissociate after activation to enter the cell nucleus and promote the transcription of target genes involved in cell survival, proliferation, and inflammation [12]. In ALS models, activation of the transcriptional factor NF- $\mathrm{KB}$ in non-neuronal cells including microglia plays a crucial pathogenic role [13]. While NF-KB-mediated transcription occurs physiologically in neurons and astrocytes [14], NF-KB activation and subsequent transcriptional activity in microglia appear as a distinctive feature of ALS [13] and other neurodegenerative conditions such as Alzheimer's disease [15]. In the ALS SOD 1 G93A mouse model, microglia-mediated motor neuron death occurs through an NF-kB-dependent mechanism and constitutive activation of NF-KB in microglia causes accelerated loss of motor neurons through a non-cell-autonomous mechanism [13]. Additionally, NF- $\mathrm{kB}$ is highly induced in microglia of sporadic ALS patients [16] and those with a mutation in optineurin, a negative regulator of TNF $\alpha$ which induces NF- $\mathrm{KB}$ activation [17]. In transgenic mice overexpressing mutant TDP-43, the protein acts as an NF-KB coactivator, and NF- $k B$ inhibition was shown to be protective [16]. Therefore, aberrant NF- $\mathrm{KB}$ activation in ALS appears to endow microglia with a neurotoxic phenotype, which could be potentially pharmacologically targeted. However, there is scarce knowledge about NF- $\mathrm{KB}$ inhibitor drugs targeting neurotoxic microglia in ALS.

Endogenous electrophilic nitroalkene fatty acids are known to target different signaling pathways including $\mathrm{Nrf} 2$ and NF-KB [18-20]. Recently, we reported the synthesis, physicochemical characterization, and therapeutic effects of nitroalkene-tocopherol analog compounds [21, 22], laying the foundation for the rational design of novel nonconventional anti-inflammatory compounds. The addition of an electrophilic nitroalkene moiety to the tocopherol scaffold confers the hybrid compound with multifaceted therapeutical activity in a model of atherosclerosis through combined inhibition of NF-KB signaling and activation of Nrf2-Keap1 [22]. In the present study, we have followed a similar approach to synthesize a hybrid compound formed by an electrophilic nitroalkene moiety coupled to benzoic acid as a scaffold. Benzoic acid is a compound widely used as a food preservative that is known to be excreted in the urine [23], and it has been used pharmacologically in large doses for the treatment of urea cycle disorders in children [24]. Also, it has been shown to exert neuroprotective effects in experimental models of Alzheimer's [25] and Parkinson's disease [26] by a complex mechanism of action that indirectly involves NF- $\mathrm{KB}$ inhibition. The lipophilic monohydroxybenzoic acid derivate, salicylic acid, also shows recognized anti-inflammatory effects, mainly through inhibition of NF-KB [27, 28]. Although benzoic and salicylic acid are currently recognized as safe compounds for human use, both require high micromolar concentrations to achieve therapeutic effects, which limit their use in the clinical setting. In this context, we reasoned that a nitroalkene benzoic acid derivative could exert anti-inflammatory effects and downregulate reactive microglia via NF-KB inhibition in a rat model of ALS bearing the SOD1 ${ }^{\mathrm{G} 93 \mathrm{~A}}$ mutation.

In the present study, we aimed to describe the synthesis, biological, and therapeutic effects of (E)-4-(2nitrovinyl)benzoic acid (BANA) in cellular and animal models of ALS. Our study focused on the BANA's potential to downregulate deleterious microglia activation through inhibition of NF-KB as compared with dimethyl fumarate, a well-known electrophilic neuroprotective marketed drug [29]. Here, we report that BANA reduced neuroinflammation and slowed disease progression in the transgenic $\mathrm{SOD} 1^{\mathrm{G} 93 \mathrm{~A}}$ rat model of ALS when administered after paralysis onset, indicating an alternative therapeutic approach to ALS.

\section{Materials and Methods}

Synthesis of (E)-4-(2-Nitrovinyl) Benzoic Acid 4Formylbenzoic acid $(4.00 \mathrm{~g}, 26.6 \mathrm{mmol})$, nitromethane 
(20 mL, $369.2 \mathrm{mmol})$, ammonium acetate $(2.55 \mathrm{~g}, 33.1 \mathrm{mmol})$, and acetic acid $(30 \mathrm{~mL})$ were sequentially introduced into a round bottom flask; then it was placed with stirring in an oil bath preheated at $90^{\circ} \mathrm{C}$ for $4 \mathrm{~h}$. The reaction mixture was then allowed to cool, the precipitate was isolated via filtration and wash with cold water. After drying the desired product was obtained as a yellow solid $(4.20 \mathrm{~g}, 82 \%)$. mp $270-272{ }^{\circ} \mathrm{C}$ (decomposition is observed). ${ }^{1} \mathrm{H}-\mathrm{NMR}$ (acetone- $\mathrm{d}_{6}$ ): $\delta=8.30$ $\left(\mathrm{d}, J=13.6 \mathrm{~Hz}, 1 \mathrm{H}, \mathrm{C} \underline{\mathrm{H}}=\mathrm{CHNO}_{2}\right) ; 8.18(\mathrm{~d}, J=13.6 \mathrm{~Hz}, 1 \mathrm{H}$, $\left.\mathrm{CH}=\mathrm{CHNO}_{2}\right) ; 8.00(\mathrm{~d}, J=8.4 \mathrm{~Hz}, 2 \mathrm{H}, \mathrm{H}-\mathrm{Ar}) ; 7.97$ (d, $J=$ $8.4 \mathrm{~Hz}, 2 \mathrm{H}, \mathrm{H}-\mathrm{Ar})$.

${ }^{13} \mathrm{C}-\mathrm{NMR}: \delta=167.1(\mathrm{C}=\mathrm{O}) ; 140.1\left(\underline{\mathrm{C}}=\mathrm{CHNO}_{2}\right) ; 138.3$ $\left(\mathrm{CH}=\underline{\mathrm{C}} \mathrm{HNO}_{2}\right) ; 134.9$ (C4-Ar); 133.8 (C1-Ar); 130.3 (C2,C3,C5,C6-Ar). MS (IE, $70 \mathrm{eV}): \mathrm{m} / \mathrm{z}(\%)=193(\mathrm{M}+, 86)$, $148\left(51, \mathrm{M}^{+}-\mathrm{CO}_{2} \mathrm{H}\right), 121\left(10, \mathrm{M}^{+}-\mathrm{CH}=\mathrm{CHNO}_{2}\right), 102$ (43: $\left.148-\mathrm{NO}_{2}\right), 91\left(85: 121-\mathrm{H}_{2} \mathrm{C}=\mathrm{O}\right), 77$ (100, Ar).

BANA Electrophilic Reactivity The electrophilic reactivity of the BANA compound was determined by analyzing the UVVisible spectra of the reaction between BANA and $\beta$ mercaptoethanol. BANA $(30 \mu \mathrm{M})$ was incubated with $\beta$ mercaptoethanol $(300 \mu \mathrm{M})$ in phosphate buffer $(20 \mathrm{mM})$ $\mathrm{pH}=7.4$, and scans were taken each minute up to $10 \mathrm{~min}$.

Sulforhodamine B Assay Cell viability was performed by the sulforhodamine B assay. Briefly, cells were placed in a 96multi-well plate and incubated with different concentrations of BANA $(5-110 \mu \mathrm{M})$ for $24 \mathrm{~h}$. After media removal, cells were washed twice with PBS $\mathrm{pH}=7.4$. Cells were fixed with trichloroacetic acid for $1 \mathrm{~h}$ at $4{ }^{\circ} \mathrm{C}$ and then washed five times with distilled water. A total of $50 \mu \mathrm{L}$ of sulforhodamine B $0.4 \% \mathrm{~m} / \mathrm{v}$ in acetic acid $1 \% \mathrm{v} / \mathrm{v}$ was added to each well and incubated $30 \mathrm{~min}$ at room temperature. After staining, the plate was washed at least five times with acetic acid $1 \% \mathrm{v} / \mathrm{v}$. Once the plate was dry the protein-bound dye was dissolved in $10 \mathrm{mM}$ Tris and the absorbance at $570 \mathrm{~nm}$ was read using a microplate spectrophotometer. The concentration which killed half of the tested cells in culture $\left(\mathrm{LC}_{50}\right)$ was determined using GraphPad Prism 7.03.

Analysis of NF-KB Activation In Vivo For NF-KB in vivo imaging studies, we used the NF-KB-RE-Luc random transgenic mouse model (Taconic, BALB/c-Tg(Rela-luc)31Xen) aged 6-8 weeks. These animals carry a transgene containing 6 NF- $k B-$ responsive elements (RE) from the CMV $\alpha$ (immediate early) promoter placed upstream of a basal SV40 promoter, and a modified firefly luciferase cDNA (Promega pGL3). The reporter is inducible by LPS and TNF $\alpha$ intraperitoneal injection [30]. Animals were randomized divided into four groups and intraperitoneally injected with increasing doses of BANA (10, 20, and $30 \mathrm{mg} / \mathrm{kg})$, benzoic acid $(30 \mathrm{mg} / \mathrm{kg})$, dimethyl fumarate $(30 \mathrm{mg} / \mathrm{kg}$ ), or vehicle (DMSO 10\% $(v / \mathrm{v}) /$ PEG 20\% (v/v) in phosphate buffer). After $2 \mathrm{~h}$, animals were intraperitoneally injected with LPS ( $1 \mathrm{mg} / \mathrm{kg})$. Differences in luciferase activity were compared between groups after $3 \mathrm{~h}$ following LPS injection. To accomplish that, $150 \mathrm{mg} / \mathrm{kg}$ of the substrate D-luciferin (\#K9918PE, XenoLight) dissolved in PBS, pH 7.4 was injected intraperitoneally to each mouse. Mice were anesthetized with isoflurane (3.0\% induction, $2.5 \%$ maintenance), placed in a ventral position in the lighttight imaging chamber, and imaged $10 \mathrm{~min}$ after luciferin injection using bioluminescence and X-ray modes ( $5 \mathrm{~s}$ acquisition, performed in Preclinical In-Vivo Xtreme II Optical/Xray imaging system, Bruker, USA). Changes in NF- $\mathrm{kB}$ activity were calculated after comparing the differences of luminescence between groups, using ImageJ software.

LPS Challenge in Mice C57BL/6 mice were randomized divided into 3 groups ( $n=3$ mice, per group) and intraperitoneally injected with BANA $(50 \mathrm{mg} / \mathrm{kg})$, benzoic acid $(50 \mathrm{mg} / \mathrm{kg})$, or vehicle (DMSO 10\% v/v in phosphate buffer). After $1 \mathrm{~h}$ animals were intraperitoneally injected with LPS $(10 \mathrm{mg} / \mathrm{kg})$ or PBS. Two hours following injection mice were sacrificed, and after peritoneal wash, blood was extracted and were both stored to measure IL-1 $\beta$ with a commercially available ELISA kit (BD OptEIA).

Study of Nuclear Translocation of NF-KB in HT-29 Reporter Cell Line The effect of BANA on nuclear translocation of NF-KB was studied by using the HT-29 NF-KB reporter cell line as previously described [31]. Briefly, cells were treated with increasing doses of BANA $(10,20$, and $30 \mu \mathrm{M})$, benzoic acid $(30 \mu \mathrm{M})$, dimethyl fumarate $(30 \mu \mathrm{M})$, or vehicle (DMSO) for $3 \mathrm{~h}$. After treatment, cells were stimulated and coincubated with TNF $\alpha$ ( $1 \mathrm{ng} / \mathrm{ml})$ for $24 \mathrm{~h}$. Finally, cells were trypsinized and resuspended for flow cytometry analysis. Cells were analyzed using an Attune NxT Flow Cytometer (Thermo Fisher Scientific) equipped with 488 and $405 \mathrm{~nm}$ lasers. Attune NxT Software was used for data acquisition and FlowJo v.10 for data analysis. Green Fluorescent Protein and propidium iodide fluorescence emissions were detected using band-pass filters 530/30 and 695/40, respectively. For each sample, 10,000 counts gated on a forward scatter versus side scatter dot plot, excluding doublets, were recorded. Only single living cells (cells that excluded propidium iodide) were considered for results comparison.

Effect of BANA on Nuclear Translocation of NF-KB in BV2 Murine Microglial Cell Line The effect of BANA on nuclear translocation of NF-KB was studied by immunocytochemistry using murine microglia cell line BV2. Cells were treated with BANA $(10,20$, and $30 \mu \mathrm{M})$, benzoic acid $(30 \mu \mathrm{M})$, or dimethyl fumarate $(30 \mu \mathrm{M})$ for $3 \mathrm{~h}$. After treatment, cells were stimulated with LPS $(100 \mathrm{ng} / \mathrm{ml})$ for $30 \mathrm{~min}$. Then, cells were washed and fixed with PFA $4 \%$ for 20 min at $4{ }^{\circ} \mathrm{C}$ and washed with PBS. Immunocytochemistry was performed as follows: 
cells were permeabilized using Triton $0.5 \%(\mathrm{v} / \mathrm{v})$ in PBS $\mathrm{pH}=7.4$ for $15 \mathrm{~min}$, and then blocked using BSA $5 \%(\mathrm{v} / \mathrm{v})$ in $\mathrm{PBS} \mathrm{pH}=7.4$ for $1 \mathrm{~h}$ at room temperature. 1:200 rabbit antiNF-kB-p65 (Abcam, \#ab16502) and 1:100 mouse antiCD11b (BD Bioscience, \#BD550219) were incubated overnight at $4{ }^{\circ} \mathrm{C}$. Then, primary antibodies were removed, washed with PBS 3 times for $10 \mathrm{~min}$, and 1:1000 Alexa Fluor-conjugated goat anti-rabbit 546/488 (Thermo Fisher Scientific, \#A11035 \#A11034) or 1:1000 goat anti-mouse 488/633 (Thermo Fisher Scientific, \#A11029 \#A21052) antibodies were incubated for $2 \mathrm{~h}$ at room temperature. After secondary antibodies were removed, cells were covered in glycerol mounting medium with 1:2000 DAPI staining. Cells were analyzed by confocal microscopy using a confocal ZEISS LSM 800. NF-kB-p65-positive nuclei were counted and the ratio NF-kB-p65/DAPI was analyzed and compared among groups. Data were analyzed using analyzing tools of ImageJ and GraphPad Prism 7.03 software.

\section{Microglia Cell Cultures from Symptomatic SOD $1{ }^{\text {G93A }}$ Rats} Primary microglial cell cultures were obtained from the spinal cord of symptomatic SOD $1^{\mathrm{G} 93 \mathrm{~A}}$ rats according to the procedures described by Trias et al. 2013 [6] with minor modifications. Briefly, animals were euthanized by overdosing with ketamine/xylazine, and the spinal cords were dissected on ice. After the meninges were removed, the spinal cord was chopped finely and dissociated with trypsin $0.25 \%(\mathrm{v} / \mathrm{v})$ in a calciumfree buffer for $5 \mathrm{~min}$ at $37^{\circ} \mathrm{C}$. Trypsin treatment was stopped by adding DMEM $/ 10 \%$ (vol/vol) Fetal Bovine Serum, in the presence of $50 \mu \mathrm{g} / \mathrm{mL}$ DNaseI and mechanical disaggregation by repeated pipetting. The resulting extract was passed through an $80-\mu \mathrm{m}$ mesh to eliminate tissue debris and then was spun. The pellet was resuspended in culture medium [DMEM/10\% (vol/ vol) Fetal Bovine Serum, Hepes (3.6 g/L), penicillin (100 IU/ $\mathrm{mL})$, and streptomycin $(100 \mu \mathrm{g} / \mathrm{mL})]$ and then was placed in a 24 multi-well culture or p35 Petri dishes. The culture medium was removed after $24 \mathrm{~h}$ and subsequently replaced every 24 or $48 \mathrm{~h}$ depending on the procedure.

\section{Nuclear Translocation Analysis of NF-KB in SOD $1{ }^{\text {G93A }}$ Adult \\ Primary Microglia The effect of BANA on nuclear transloca-} tion of NF-KB was studied by immunocytochemistry using SOD $1^{\mathrm{G} 93 \mathrm{~A}}$ primary microglia cells. Cells were treated with BANA $(10,20$, and $30 \mu \mathrm{M})$, benzoic acid $(30 \mu \mathrm{M})$, or dimethyl fumarate $(30 \mu \mathrm{M})$ for $3 \mathrm{~h}$. After treatment, cells were stimulated with LPS $(100 \mathrm{ng} / \mathrm{ml})$ for $30 \mathrm{~min}$. Then, cells were washed and fixed with PFA $4 \%$ for $20 \mathrm{~min}$ at $4{ }^{\circ} \mathrm{C}$ and washed with PBS. Immunocytochemistry was performed as follows: cells were permeabilized using Triton $0.5 \%(\mathrm{v} / \mathrm{v})$ in PBS $\mathrm{pH}=7.4$ for $15 \mathrm{~min}$, and then blocked using BSA 5\% (v/v) in $\mathrm{PBS} \mathrm{pH}=7.4$ for $1 \mathrm{~h}$ at room temperature. 1:200 rabbit antiNF-kB-p65 (Abcam, \#ab16502) and 1:100 mouse antiCD11b (BD Bioscience, \#BD550219) were incubated overnight at $4{ }^{\circ} \mathrm{C}$. Then, primary antibodies were removed, washed with PBS 3 times for $10 \mathrm{~min}$, and 1:1000 Alexa Fluorconjugated goat anti-rabbit 488 (Thermo Fisher Scientific, \#A11034) or 1:1000 goat anti-mouse 633 (Thermo Fisher Scientific, \#A21052) antibodies were incubated for $2 \mathrm{~h}$ at room temperature. After secondary antibodies were removed, cells were covered in glycerol mounting medium with 1:2000 DAPI staining. Cells were analyzed by confocal microscopy using a confocal ZEISS LSM 800. NF-kB-p65-positive nuclei were counted and the ratio NF-kB-p65/DAPI was analyzed and compared among groups. Data were analyzed using analyzing tools of ImageJ and GraphPad Prism 7.03 software.

\section{Analysis by RT-qPCR of NF-KB-Dependent Gene Expression} The effect of BANA or benzoic acid over NF-kB-dependent gene expression was studied by RT-qPCR in SOD $1^{\text {G93A }}$ adult primary microglia cells. Cells were treated with BANA (10, 20 , and $30 \mu \mathrm{M})$, benzoic acid $(30 \mu \mathrm{M})$, or dimethyl fumarate $(30 \mu \mathrm{M})$ for $3 \mathrm{~h}$. After treatment, cells were stimulated with LPS (100 ng/mL, $30 \mathrm{~min})$. After harvesting the cells, mRNA extraction using the RNeasy Micro Kit (QIAGEN) was performed according to the manufacturer's instructions. mRNA yields were measured on Nanodrop device (Thermo Fisher Scientific) and cDNA was obtained from $0.5 \mu \mathrm{g}$ of RNA, $4 \mu \mathrm{L}$ of iScript reverse transcription Supermix for RT-qPCR (Bio-Rad, $-20{ }^{\circ} \mathrm{C}$ ) in a final volume of $20 \mu \mathrm{L}$ filled with nuclease-free water. The Thermocycler was set as follows: priming $5 \mathrm{~min}$ at $25{ }^{\circ} \mathrm{C}$ followed by $30 \mathrm{~min}$ at $42{ }^{\circ} \mathrm{C}$ for reverse transcription and $5^{\prime}$ at $85^{\circ} \mathrm{C}$ for RT inactivation. RT-qPCR was performed on reverse-transcribed cDNA using SsoAdvanced ${ }^{\mathrm{TM}}$ Universal SYBR ${ }^{\circledR}$ Green Supermix (BioRad) on a CFX96 Touch ${ }^{\mathrm{TM}}$ real-time PCR detection system. For each well, $5 \mu \mathrm{L}$ of diluted DNA was added to $20 \mu \mathrm{L}$ of the mix (containing $1 \mu \mathrm{L}$ of each primer, $12.5 \mu \mathrm{L}$ of SsoAdvanced ${ }^{\mathrm{TM}}$ Universal SYBR ${ }^{\circledR}$ Green Supermix, $5.5 \mu \mathrm{L}$ of nuclease-free water). Each sample was run in duplicate. The cycling parameters were as follows: $30 \mathrm{~s}$ at $95^{\circ} \mathrm{C}$ then 40 cycles at $95{ }^{\circ} \mathrm{C}$ for 10 and $30 \mathrm{~s}$ at $60{ }^{\circ} \mathrm{C}$. Cq values were obtained for every cycle. Primers were designed on NCBI Primer-BLAST following the best guidelines to exclude genomic DNA amplification. The analysis was done using BioRad CFX manager 3.1 with a threshold set at 650 RFU corresponding to the amplification curves linear portion. Variations between samples were normalized using two housekeeping genes $\beta$-Actin and HPRT. All primers were validated for specificity and efficiency. The following rat primers were used:

TNF- $\alpha$ forward: 5 '- ATC CGA GAT GTG GAA CTG $\mathrm{GC}-3^{\prime}$;

TNF- $\alpha$ reverse: $5^{\prime}$ - TGG GAA CTT CTC CTC CTT GTT G -3'; 
IL-6 forward: 5'- TTC TCT CCG CAA GAG ACT TCC $3^{\prime}$;

IL-6 reverse: 5'- TCT CCT CTC CGG ACT TGT GAA $3^{\prime}$;

MCP-1 forward: 5'- TGT CTC AGC CAG ATG CAG TTA AT - $3^{\prime}$;

MCP-1 reverse: 5'- TCC AGC CGA CTC ATT GGG AT $-3^{\prime}$.

IL-1 $\beta$ forward: 5'- TAG CAG CTT TCG ACA GTG AGG -3';

IL- $1 \beta$ reverse: $5^{\prime}$ - CTC CAC GGG CAA GAC ATA GG $-3^{\prime}$;

HPRT forward: 5'- GTC ATG TCG ACC CTC AGT CC $-3^{\prime}$.

HPRT reverse: 5'- GCA AGT CTT TCA GTC CTG TCC 3';

Animals Male SOD $1^{\mathrm{G} 93 \mathrm{~A}}$ progeny were used for further breeding to maintain the line. Rats were housed in a centralized animal facility with a $12 \mathrm{~h}$ light-dark cycle with ad libitum access to food and water. Perfusion with fixative was performed under $90 \%$ ketamine- $10 \%$ xylazine anesthesia and all efforts were made to minimize animal suffering, discomfort, or stress. All procedures using laboratory animals were performed under the national and international guidelines and were approved by the Institutional Animal Committee for animal experimentation (CEUA Approved protocol: \#005-17 to Dr. Luis Barbeito). This study was carried out in strict accordance with the Institut Pasteur de Montevideo Committee's requirements and under the current ethical regulations of the Uruguayan Law $\mathrm{N}^{\circ} 18.611$ for animal experimentation that follow the Guide for the Care and Use of Laboratory Animals of the National Institutes of Health (USA).

Immunohistochemical Staining of Rats' Spinal Cords At least 4 rats were analyzed for each immunohistochemistry experiment. Three different conditions were studied as follows: 1) non-transgenic (NonTg) rats of 160-180 days; 2) transgenic SOD $1^{\mathrm{G} 93 \mathrm{~A}}$ rats of $195-210$ days treated with vehicle (paralysis, 15d-vehicle) and 3) transgenic $\mathrm{SOD} 1^{\mathrm{G} 93 \mathrm{~A}}$ rats of 195-210 days treated with 50 or $100 \mathrm{mg} / \mathrm{kg} /$ day of BANA during 15 days. After treatment animals were deeply anesthetized and transcardial perfusion was performed with paraformaldehyde 4\% (v/v) in PBS $\mathrm{pH}=7.4$. Fixed spinal cords were removed, post-fixed by immersion overnight in paraformaldehyde 4\% (v/v) in PBS pH = 7.4, and then transverse sectioned $(30 \mu \mathrm{m})$ in a Leica cryostat. Serial sections were collected in PBS $\mathrm{pH}=7.4$ for immunohistochemistry. Free-floating sections were blocked and permeabilized for $1 \mathrm{~h}$ at room temperature with Triton X-100 0.5\% (v/v)/BSA 5\% (v/v) in PBS $\mathrm{pH}=7.4$, passed through washing buffered solutions, and incubated overnight at $4{ }^{\circ} \mathrm{C}$ in a solution of Triton X-100 $0.5 \%$ $(\mathrm{v} / \mathrm{v}) / \mathrm{BSA} 1 \%(\mathrm{v} / \mathrm{v})$ in $\mathrm{PBS} \mathrm{pH}=7.4$ containing the following primary antibodies: 1:500 rabbit anti-Iba1 (Wako, \#01919741), 1:300 mouse anti-Iba1 (Millipore, \#MABN92), 1:300 rabbit anti-ChAT (Millipore, \#AB143), 1:300 mouse anti-CD68 (Abcam, \#ab31630), 1:200 rabbit anti-CD34 (Abcam, \#ab81289), 1:400 mouse anti-GFAP (Sigma, \#G3893), 1:400 rabbit anti-GFAP (Sigma, \#G9269), 1:400 mouse anti-S100ß (Sigma, \#S2532), 1:200 rabbit antiNF-kB-p65 (Abcam, \#ab16502), 1:200 rabbit anti-Ki67 (Abcam, \#ab16667), 1:200 rabbit anti-ubiquitin (Millipore, \#05-1307), 1:200 mouse anti-nitrotyrosine (Millipore, \#05233), 1:300 anti-VGlut-1 (SySy, \#135-303), 1:300 antisynaptophysin (Abcam, \#206870), 1:200 anti- $\beta$ III-Tubulin (Millipore, \#MAB1637). Immunostaining with rabbit antiNF-kB-p65, mouse anti-Iba1, rabbit anti-Ki67, mouse anti$\mathrm{CD} 68$, and rabbit anti-ubiquitin required free-floating citrate buffer (sodium citrate $10 \mathrm{mM}, \mathrm{pH}$ 6) antigen retrieval at $95^{\circ} \mathrm{C}$ for $5 \mathrm{~min}$. NeuroTrace 530/615 red fluorescent Nissl stain (Thermo Fisher Scientific, \#B34650) was also used for visualizing neurons. After incubation with primary antibodies, free-floating slices were washed with PBS 3 times for $10 \mathrm{~min}$, incubated for $2 \mathrm{~h}$ at room temperature with the following secondary antibodies: 1:500 goat anti-rabbit-Alexa Fluor 488 (Thermo Fisher Scientific, \#A21052), 1:500 goat anti-mouse-Alexa Fluor 546 (Thermo Fisher Scientific, \#A11035), 1:500 goat anti-mouse-Alexa Fluor 633 (Thermo Fisher Scientific, \#A21052), 1:500 Streptavidin-Alexa Fluor 405 or Alexa Fluor 633 (Thermo Fisher Scientific, \#S21375), washed with PBS 3 times for $5 \mathrm{~min}$ and mounted in DPX mounting medium (Sigma, \#06522-100ML).

Analysis of Gliosis in the Ventral Horn of the Lumbar Spinal Cord Microgliosis and astrogliosis were assessed by measuring the expression intensity for the different markers in the gray matter of the lumbar spinal cord of non-transgenic and symptomatic SOD $1^{\mathrm{G} 93 \mathrm{~A}}$ rats that were treated with either vehicle or BANA. Microgliois was assessed by the analysis of different microglia typical markers such as Iba1, CD68, CD34, and Isolectin. The number of aberrant glial cells coexpressing the astrocytic markers GFAP and $\mathrm{S} 100 \beta$ was assessed by counting the respective positive cells for both markers in gray matter from the lumbar cord among groups as previously described [5]. All analyses were performed in at least 10 histological sections per animal (3-5 different rats for each condition) using the ImageJ software.

Analysis of Motor Neuron Number and Size The number of motor neurons expressing ChAT was assessed by counting the positive cells in the gray matter of the lumbar spinal cord of non-transgenic compared with symptomatic SOD $1^{\mathrm{G} 93 \mathrm{~A}}$, vehicle- and BANA-treated rats. Motor neuron counting was based on a stereological approach as previously reported [32]. Briefly, ChAT-positive cells were quantified on ten $30 \mu \mathrm{m}$ sections taken $300 \mu \mathrm{m}$ apart from the ventral horn, comparing 
the cell numbers in both Rexed laminae VII and IX, which display low and high density of large motor neurons, respectively. The longest axis (length) of each soma was taken into consideration to quantify the mean size of motor neuron soma. The analysis was performed manually in at least 25 histological sections per animal (4 different rats for each condition) using the cell counter tool of the ImageJ software.

Analysis of Synaptic Terminals Contacting Motor Neuron in the Spinal Cord The number of synaptic vesicles contacting motor neuron cell bodies was assessed by immunohistochemistry by counting VGlut-1-positive and synaptophysin-positive puncta in Rexed laminae VII and IX. Synaptic terminals were quantified in at least 20 motor neurons per animal (4 different rats per condition) using the Analyze Particles tool of ImageJ software.

\section{Immunohistochemistry of Whole-Mounted Muscle and} Neuromuscular Junction Innervation Analysis As previously described [33], extensor digitorium longus (EDL) muscles from the NonTg and SOD1 ${ }^{\mathrm{G} 93 \mathrm{~A}}$ hind limbs were dissected. Then, tissues were blocked for $2 \mathrm{~h}$ at room temperature (BSA 5\% $\mathrm{v} / \mathrm{v} /$, Triton X-100 0.8\% v/v in PBS $\mathrm{pH}=7.4$ ), incubated with primary antibodies or fluorescent probes at $4{ }^{\circ} \mathrm{C}$ overnight: axon and postsynaptic plates were carried out using 1:1000 rabbit antiheavy chain neurofilament-Alexa Fluor 555 (Millipore, \#MAB5256A5) and fluorescently labeled $\alpha$-bungarotoxinFITC $[\alpha B T X$, (Thermo Fisher Scientific, B13422)], axon presynaptic terminals were labeled with 1:300 rabbit antisynaptophysin-Alexa Fluor 555 (Abcam, \#ab206870). After washing 4 times with PBS, whole-mount muscles were mounted using DPX. Structural changes of the neuromuscular junction were scored using maximum intensity projections of images acquired from whole-mounted muscles. Briefly, neuromuscular junction innervation analysis was performed taking into consideration those postsynaptic motor endplates occupied by a presynaptic axon terminal, where full innervation was defined as at least $80 \%$ of overlapping between pre- and postsynaptic. An average of 100 neuromuscular junctions per animal was analyzed using the ImageJ software.

Human Tissue Collection The collection of postmortem human ALS and control samples was approved by the University of Alabama, Birmingham (UAB) Institutional Review Board (Approved IRB Protocol: X091222037 to Dr. Peter H. King). All ALS patients were cared for at UAB and so detailed clinical records were available. Control samples were age-matched and were harvested from patients who expired from non-neurological causes. The average collection time after death was less than $10 \mathrm{~h}$. All tissues were harvested by PHK and YS at the time of autopsy and preserved within $30 \mathrm{~min}$.

Human Spinal Cord Immunohistochemistry As previously described [34], 10- $\mu \mathrm{m}$ spinal cord paraffin sections were sliced using a microtome. Following deparaffinization, slices were blocked and permeabilized in BSA 5\% (v/v)/Triton X-100 0.5\% $(\mathrm{v} / \mathrm{v})$ for $2 \mathrm{~h}$ at room temperature. Previously described primary antibodies were incubated in BSA $1 \%(\mathrm{v} / \mathrm{v}) /$ Triton X-100 0.5\% (v/v) in PBS $\mathrm{pH}=7.4$ at $4{ }^{\circ} \mathrm{C}$ overnight. After washing, secondary antibodies were incubated for $3 \mathrm{~h}$ at room temperature. After PBS washing, Mowiol medium (Sigma, St. Louis, MO, USA) was used for mounting. Only ventral lumbar spinal cord sections were analyzed. Motor neuron somas were identified in the ventral spinal cord by typical morphology and nuclei.

Fluorescence Imaging Fluorescence imaging was performed with a laser scanning Zeiss LSM 800 or LSM 880 confocal microscope with either a $\times 25$ (1.2 numerical aperture) objective or $\times 63$ (1.3 numerical aperture) oil immersion objective using Zeiss Zen Black software. Maximum intensity projections of optical sections were created with Zeiss Zen software. Maximum intensity projections of optical sections, as well as $3 \mathrm{D}$ reconstructions, were created with Zeiss Zen software.

BANA Post-paralysis Survival Trial The preclinical trial was performed as previously described with modifications [8]. To

Fig. 1 BANA inhibits NF-KB activation and transcription. (a) A synthesis scheme of (E)-4-(2-nitrovinyl) benzoic acid (BANA, red). (b) BANA inhibits NF- $\mathrm{kB}$ activation in NF-kB-RE-Luc transgenic mice. BANA, Benzoic acid (BA), or dimethyl fumarate (DMF) were administered $2 \mathrm{~h}$ before intraperitoneal injection of LPS. The graph shows the quantitative analysis in different experimental conditions. All quantitative data are expressed as mean \pm SEM; data were analyzed by Ordinary one-way ANOVA followed by Tukey's multiple comparisons test, ***p= $0.0009 * * * * p<0.0001 . n=4$ animals per condition. (c) Confocal images of BV2 microglia treated with BANA, BA, or DMF before LPS stimulation. BANA inhibits LPS-induced NF-kB-p65 nuclear translocation. Cells in the sham condition were not treated with LPS. Nuclear NF-KBp65 colocalizing with DAPI nuclear staining is denoted in yellow. The graph shows the quantitative analysis of the ratio nuclear NF-kB-p65/ DAPI among experimental conditions. All quantitative data are expressed as mean \pm SEM; data were analyzed by Ordinary one-way ANOVA followed by Tukey's multiple comparisons test, $* p=0.0148 * * p=$ $0,0031 * * * * p<0,0001.3$ replicates of 3 independent experiments. Scale bar $=10 \mu \mathrm{m}$. (d) Confocal images show CD11b-positive SOD $1^{\text {G93A }}$ primary microglia (gray) treated with BANA, BA or vehicle, before LPS stimulation. BANA inhibits LPS-induced NF-kB-p65 nuclear translocation in SOD1 ${ }^{\mathrm{G} 93 \mathrm{~A}}$ microglia. Nuclear NF-kB-p65 colocalizing with DAPI nuclear staining is denoted in yellow. The graph shows the quantitative analysis of the ratio nuclear NF- $\mathrm{kB}-\mathrm{p} 65 / \mathrm{DAPI}$ among experimental conditions. All quantitative data are expressed as mean $\pm \mathrm{SEM}$; data were analyzed by Ordinary one-way ANOVA followed by Tukey's multiple comparisons test, $* * * * p<0.0001,3$ replicates of 4 independent experiments. Scale bar $=20 \mu \mathrm{m}$. (e) Analysis by RT-qPCR of mRNA levels of NF-kB-associated proinflammatory cytokines CCL2, IL-6, IL$1 \beta$, and TNF $\alpha$ following LPS stimulation in SOD $1^{\mathrm{G} 93 \mathrm{~A}}$ primary microglia. BANA prevents NF-KB-mediated gene transcription induced by LPS. Data are expressed as mean \pm SEM; data were analyzed by Ordinary oneway ANOVA followed by Tukey's multiple comparisons test, *p = $0,0395(\mathrm{CCL} 2) * p=0.0409$ (IL-6) $* * p=0.0049$ (IL-1 $\beta) * p=0.0292$ $* * p=0.0063(\mathrm{TNF} \alpha) .2$ replicates of 3 independent experiments 
a

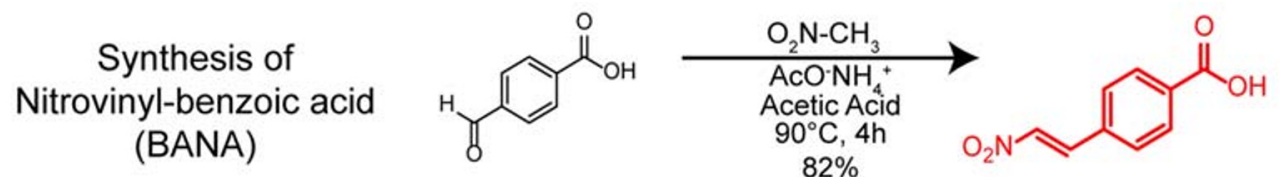

(BANA)

$82 \%$
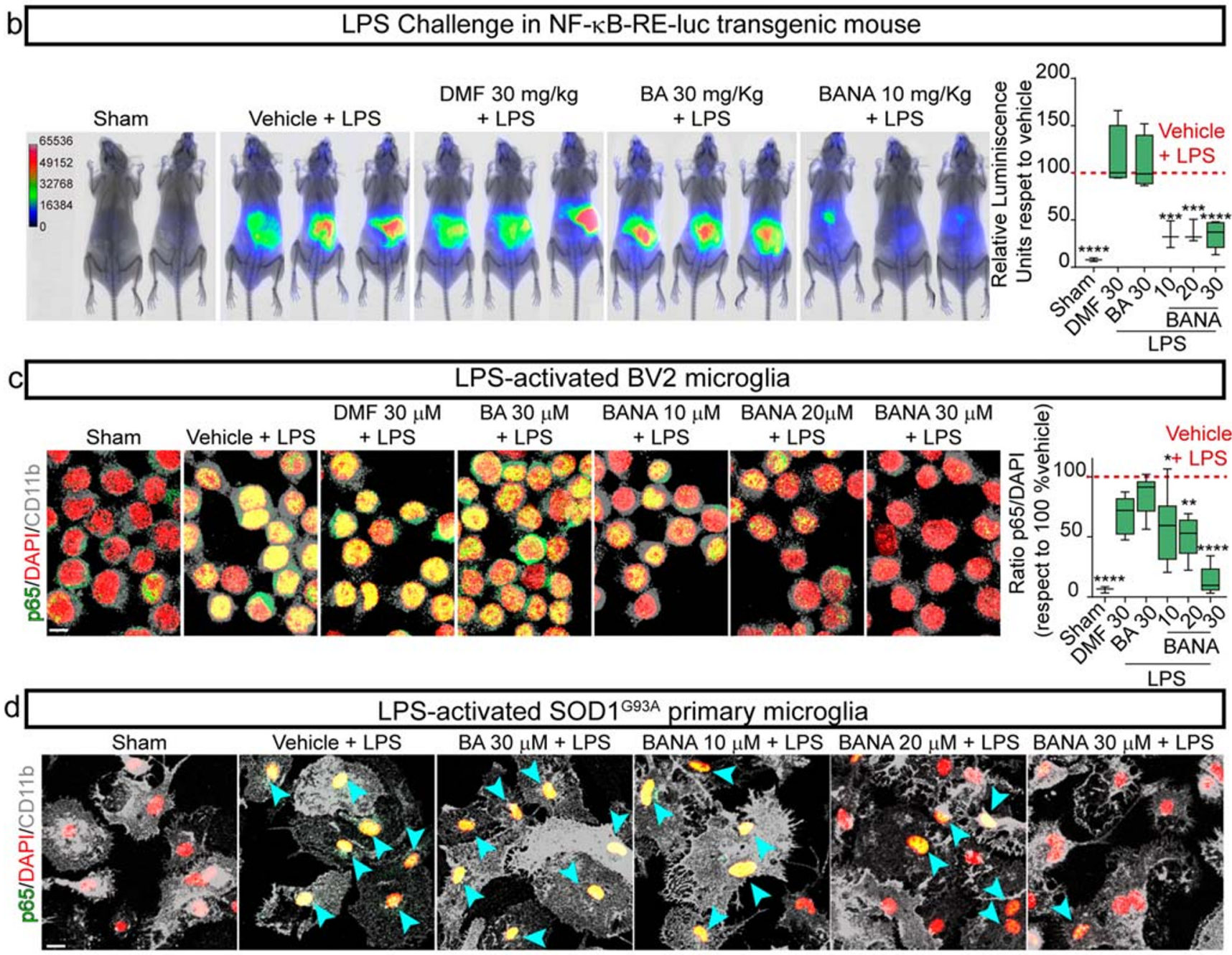

LPS-activated SOD1 ${ }^{\text {G93A }}$ primary microglia
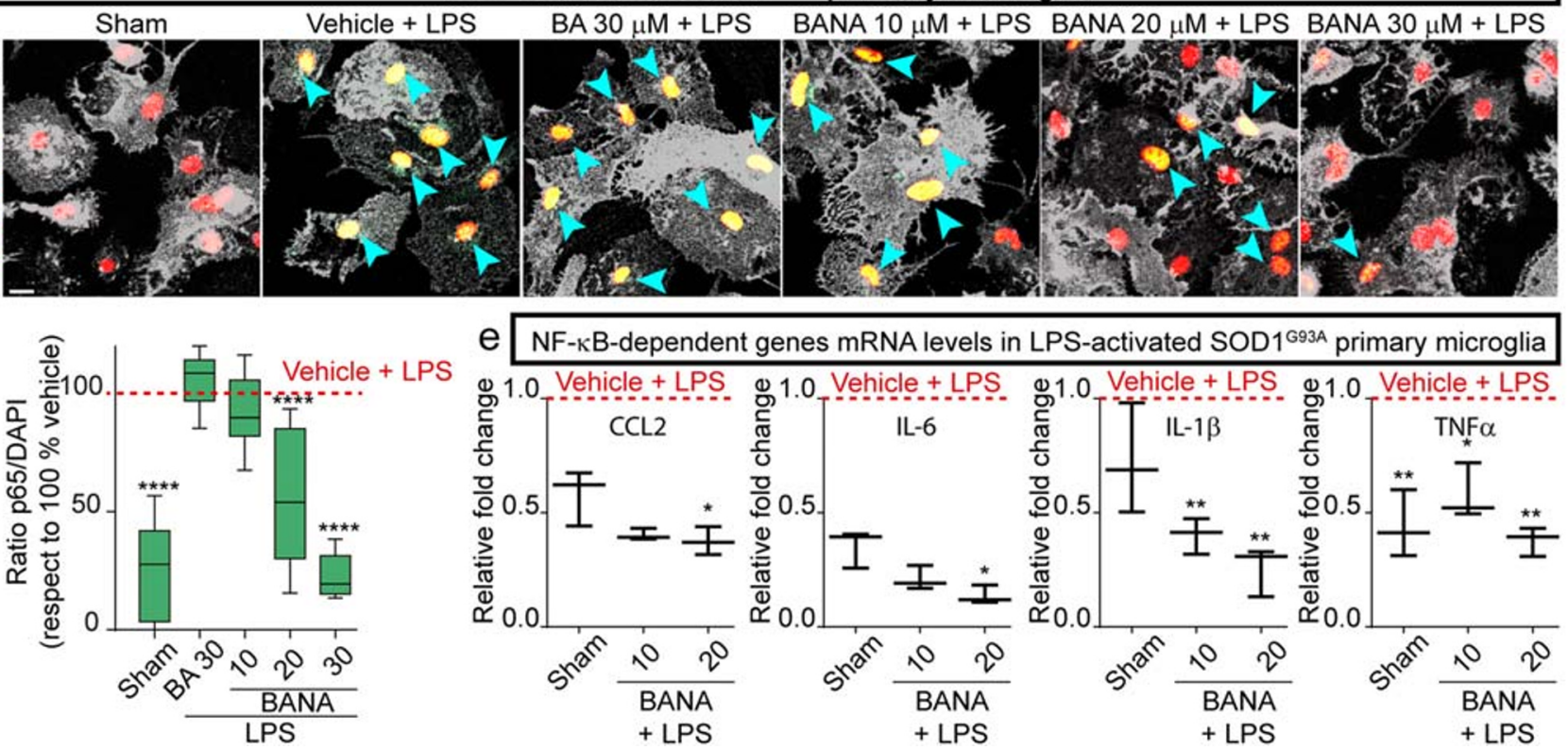

e NF-KB-dependent genes mRNA levels in LPS-activated SOD ${ }^{693 A}$ primary microglia
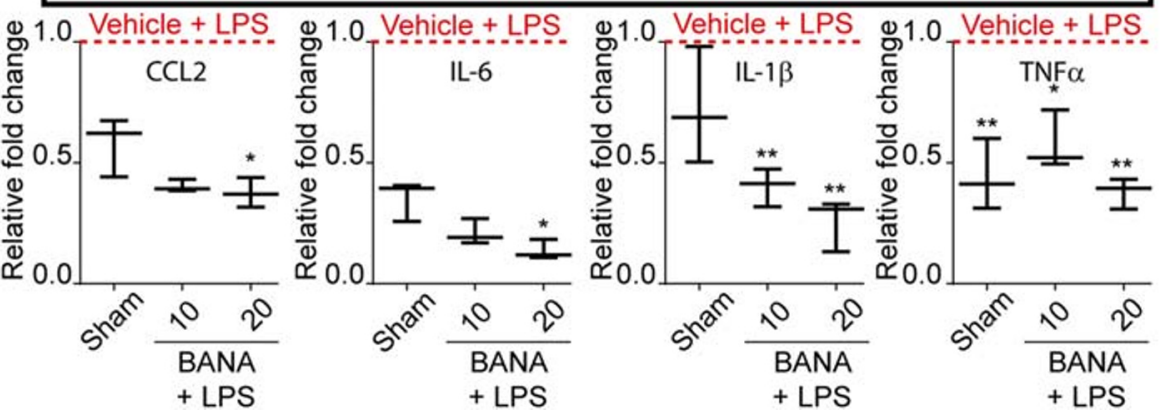

reduce sex variability, only female transgenic SOD $1^{\mathrm{G} 93 \mathrm{~A}}$ rats showing weakness and gait alterations in the hind limbs as the first clinical sign were selected for BANA treatment studies. Rats were divided randomly into BANA or vehicle-treated groups. A total of 10 female rats were assigned to each experimental group. BANA was freshly prepared in phosphate buffer, and administrated daily at a dose of $100 \mathrm{mg} / \mathrm{kg}$ using a curved stainless steel gavage needle with a 3-mm ball tip. Rats were treated from day-1 after paralysis onset until the end stage when they were euthanized. 
a
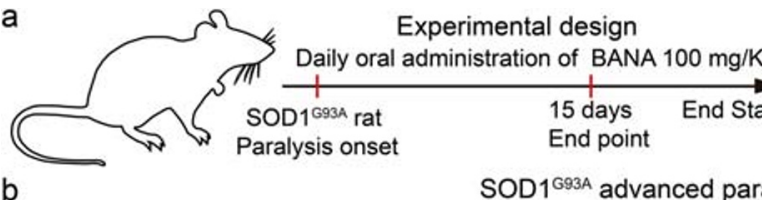

Paralysis onset End point

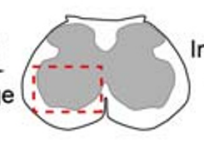

Immunohistological analysis

of the ventral horn of the

lumbar spinal cord

b SOD1 ${ }^{\text {G93A }}$ advanced paralysis
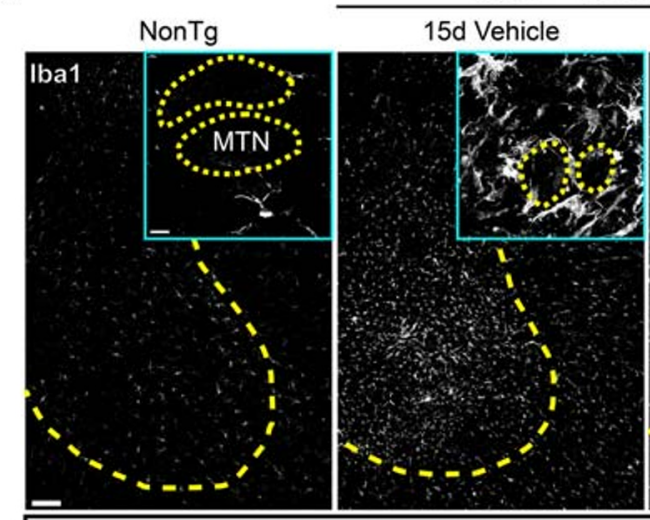

15d BANA

C

Nuclear expression of NF-кB-p65 in the spinal cord from SOD1 ${ }^{\mathrm{G} 93 \mathrm{~A}}$ rats
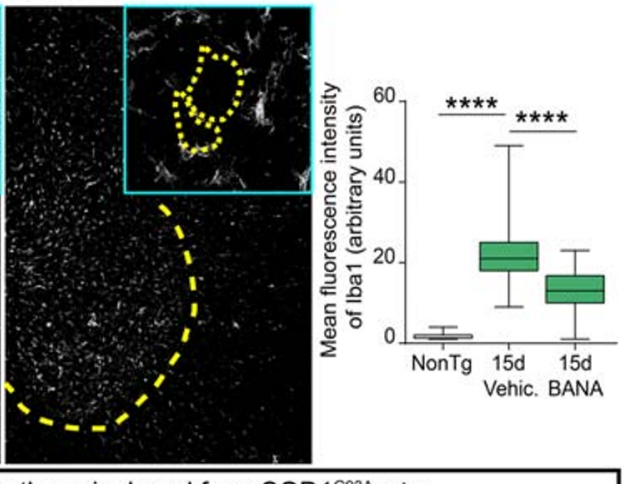

NonTg

SOD $1^{\text {G93A }}$ advanced paralysis
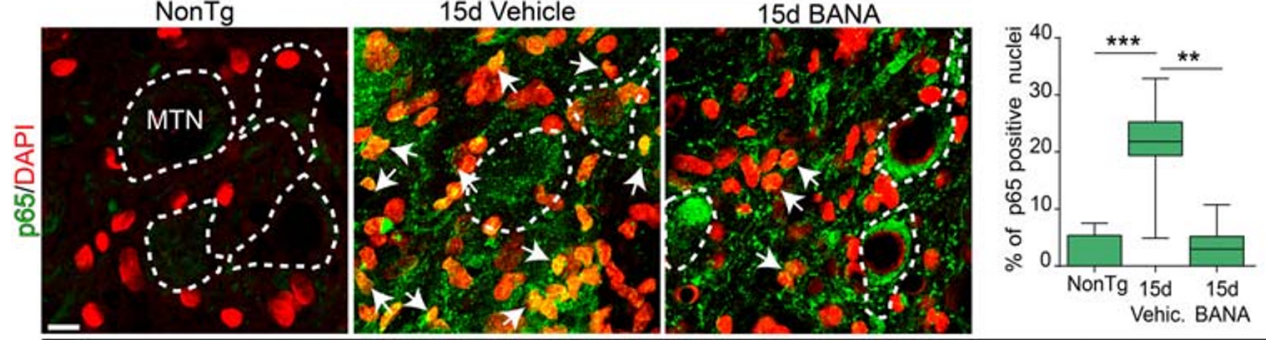

d Nuclear expression of NF-KB-p65 in spinal cord microglia form SOD1 ${ }^{\text {G93A }}$ advanced paralysis vehicle

p65/Nissl

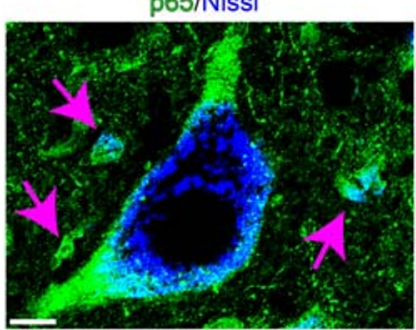

p65/Nissl/DAPI

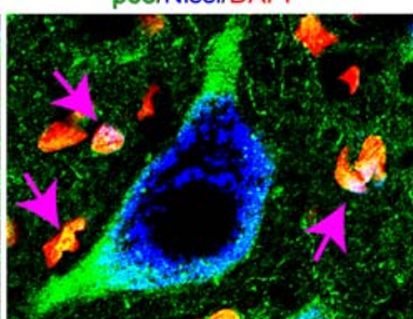

p65/Nissl/DAPI/leolsciin

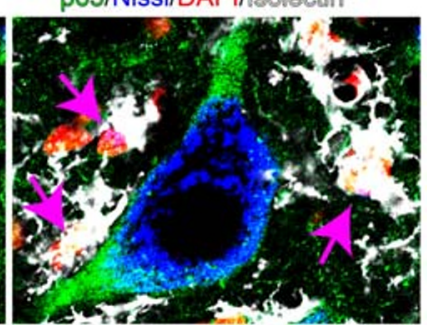

Fig. 2 Post-paralysis treatment with BANA $(100 \mathrm{mg} / \mathrm{Kg})$ reduces microgliosis and prevents microglial NF-KB-p65 nuclear translocation in the spinal cord of SOD1 $1^{\mathrm{G} 93 \mathrm{~A}}$ rats. (a) Scheme of the experimental design. SOD $1^{\mathrm{G} 93 \mathrm{~A}}$ female rats were treated with $100 \mathrm{mg} / \mathrm{Kg} /$ day of BANA or vehicle immediately after the first signs of paralysis onset of one hindlimb and continue for 15 days. Immunohistological analysis of the lumbar spinal cord of nontransgenic and vehicle- and BANA-treated SOD1 ${ }^{\mathrm{G} 93 \mathrm{~A}}$ symptomatic rats were performed. (b) Confocal images showing Iba1-positive microglia (white) in the ventral spinal cord. Yellow dotted lines delimit white from gray matter. Insets show microgliaassociated motor neurons (MTN, yellow dotted lines). Note that postparalysis treatment with BANA significantly reduced microgliosis after 15 days as well as the number of hypertrophic microglia that surround motor neurons in the ventral spinal cord. The graph shows a quantitative analysis of microgliosis in the ventral spinal cord. Data are expressed as mean \pm SEM; data were analyzed by the Kruskal-Wallis test followed by
Dunn's multiple comparisons test, $* * * * p<0.0001 . n=4$ animals per condition. Scale bars $=100 \mu \mathrm{m}, 10 \mu \mathrm{m}$ (inset). (c) Confocal images show NF-kB-p65 (green) and DAPI (red) expression in the ventral horn of the spinal cord of nontransgenic and symptomatic SOD $1^{\mathrm{G} 93 \mathrm{~A}}$ rats, treated with vehicle or BANA, in the surroundings of motor neurons (white dotted lines). Nuclei stained in yellow denote nuclear NF-kB-p65 and DAPI colocalization (white arrows). The graph to the right shows the quantitative analysis of the ratio NF-kB-p65/DAPI in the surroundings of motor neurons. Data are expressed as mean $\pm \mathrm{SEM}$; data were analyzed by the Kruskal-Wallis test followed by Dunn's multiple comparisons test, $* * p=0.0018, * * * p=0.0003 . n=4$ animals per condition. Scale bar $=20 \mu \mathrm{m}$. (d) High magnification confocal images showing NF- $\kappa B-$ p65 nuclear expression in Isolectin-positive microglia (white) surrounding degenerating motor neurons stained with Nissl (blue) in the symptomatic SOD1 ${ }^{\mathrm{G} 93 \mathrm{~A}}$ ventral spinal cord. Scale bar $=10 \mu \mathrm{m}$
Determination of Disease Onset and End-Stage All rats were weighed and evaluated for motor activity daily. Disease onset was determined for each animal when pronounced muscle atrophy accompanied by an abnormal gait, typically expressed 
as subtle limping or dragging of one hind limb. The end stage was defined by a lack of righting reflexes or the inability to reach food and water.

Determination of BANA Plasma Levels For the detection of BANA in plasma, we performed an RP-HPLC analysis (Agilent 1200 HPLC system). SOD $1^{\mathrm{G} 93 \mathrm{~A}}$ rats were treated with $100 \mathrm{mg} / \mathrm{kg} /$ day BANA or vehicle for 15 days. After treatment rats were sacrificed and plasma samples were obtained. $100 \mu \mathrm{L}$ of plasma was incubated with $\mathrm{HgCl}_{2}(20 \mathrm{mM})$ at $37{ }^{\circ} \mathrm{C}$ for $30 \mathrm{~min}$. Then, extraction with $900 \mu \mathrm{L}$ of acetonitrile (ACN) was performed and injected into HPLC in a 20 $40 \%$ gradient of B phase for 10 min. BANA plasma concentration was calculated from a standard curve using salicylate as an internal standard. (CPAK C- $18,4 \mu \mathrm{M}, 150 \mathrm{~mm} \times$ $3.9 \mathrm{~mm}$ I.D. column; A phase: $\mathrm{H}_{2} \mathrm{O} 0.1 \%$ formic acid; B phase: ACN $0.1 \%$ formic acid; detection at $300 \mathrm{~nm}$ ).

Statistics Analysis Quantitative data were expressed as mean \pm SEM. Ordinary one-way ANOVA followed by Tukey's multiple comparisons test, Kruskal-Wallis followed by Dunn's multiple comparisons test, Mann-Whitney comparisons test, or unpaired $t$ test were used for statistical analysis, with $p<0.05$ considered significant. The GraphPad Prism 7.03 software was used for statistical analyses. All selected images represent the mean value for each condition.

\section{Results}

\section{BANA Inhibits NF-KB Activation Induced by LPS and TNFa As} shown in Fig. 1a, BANA was obtained in a one-step synthetic route by condensation of commercially available $p$ formylbenzoic acid with nitromethane, in the presence of ammonium acetate. The analysis of ${ }^{1} \mathrm{H}$ NMR and ${ }^{13} \mathrm{CNMR}$ spectra confirmed that only one stereoisomer was formed (Fig. S1a and $b$ ). Due to the coupling constant of the doublets of the alkene observed in the ${ }^{1} \mathrm{H}$ NMR spectra (Fig. S1a), the configuration of the nitroalkene was consistent with an $E$ isomer. As previously shown for other endogenous or synthetic nitroalkene compounds [21], BANA also displayed electrophilic properties as shown by its ability to react via the Michael addition reaction with nucleophiles such as $\beta$ mercaptoethanol (Fig. S1c).

To determine whether BANA was capable of preventing LPS-induced NF- $\mathrm{KB}$ activation, intraperitoneal administration of BANA $(10,20$, and $30 \mathrm{mg} / \mathrm{Kg})$ were followed by LPS intraperitoneal administration to an NF-KB-reporter transgenic mouse. Its scaffold benzoic acid $(30 \mathrm{mg} / \mathrm{Kg})$ and dimethyl fumarate $(30 \mathrm{mg} / \mathrm{Kg})$, an electrophilic drug currently assayed in ALS clinical trials [35], were used as controls. BANA treatment significantly decreased NF- $\kappa B$ activation pathway at all studied doses (Fig. 1b and Fig. S2). In comparison, the benzoic acid scaffold $(30 \mathrm{mg} / \mathrm{Kg})$ and the electrophilic drug dimethyl fumarate $(30 \mathrm{mg} / \mathrm{kg})$ were devoid of inhibitory effect in NF- $\mathrm{KB}$ pathways in this model. In turn, BANA was able to reduce IL- $1 \beta$ concentration in plasma and peritoneum of mice intraperitoneally injected with LPS with respect to controls (Fig. S3).

The effects of BANA on LPS- or TNF $\alpha$-induced NF- $\mathrm{KB}$ activation were also assayed in cell cultures of BV2 microglial cell line and primary adult microglia isolated from symptomatic SOD $1^{\mathrm{G} 93 \mathrm{~A}}$ rats, and HT-29 NF-kB reporter cell line, respectively. As shown in Fig. 1c and d and Fig. S4, BANA $(10-30 \mu \mathrm{M})$ potently prevented NF-KB activation as assessed by either LPS-induced NF-kB-p65 nuclear translocation in $\mathrm{BV} 2$ cells and SOD ${ }^{\mathrm{G} 93 \mathrm{~A}}$ microglia or TNF $\alpha$-induced NF-kB-p65 nuclear translocation in HT-29 NF- $k B$ reporter cell line. In addition, BANA prevented LPS-induced upregulation of NF-kB-dependent transcriptional activity of MCP1, IL-6, IL- $1 \beta$, and TNF $\alpha$ assessed by RT-qPCR in adult microglia isolated from symptomatic SOD1 ${ }^{\mathrm{G} 93 \mathrm{~A}}$ rats (Fig. 1e). The benzoic acid scaffold and dimethyl fumarate were devoid of inhibitory effect in NF-KB pathways in these experimental settings. The $\mathrm{LC}_{50}$ values assessed in cell cultures were $50 \mu \mathrm{M}, 30 \mu \mathrm{M}$, and $80 \mu \mathrm{M}$, for BV2, SOD $1^{\mathrm{G} 93 \mathrm{~A}}$ adult microglia, and HT-29, respectively.

Post-paralysis Treatment with BANA in SOD $1{ }^{\mathrm{G} 93 \mathrm{~A}}$ Rats Abrogates NF-KB Activation in Microglia Next, we assessed the effect of BANA on markers of neuroinflammation in the degenerating spinal cords. First, dosing with $100 \mathrm{mg} /$ $\mathrm{Kg} / \mathrm{day}$ of BANA administered orally to rats was defined based on previous reports showing antineuroinflammatory effects and safety of benzoic acid at the same concentration [36]. Moreover, BANA plasma levels reached $3.49 \mu \mathrm{M}$ as assessed by HPLC following $100 \mathrm{mg} / \mathrm{Kg} /$ day dosing (Table S1), a concentration considered within the expected therapeutic range.

$\mathrm{SOD} 1{ }^{\mathrm{G} 93 \mathrm{~A}}$ rats were treated daily with BANA $(100 \mathrm{mg} / \mathrm{kg})$ or vehicle at the onset of motor symptoms as previously described [8]. After 15 days of treatment, rats were euthanized and spinal cords were dissected for immunohistological analysis. Compared to vehicle-treated rats, post-paralysis treatment with BANA significantly reduced microgliosis in the ventral horn of the spinal cord as assessed by Iba1-, CD68-, and CD34-positive microglial cells (Fig. 2b and Fig. S5). In addition, post-paralysis treatment with BANA significantly reduced astrocytosis assessed by GFAP staining as well as the emergence of aberrant glial cells characterized by the double staining GFAP/S100 $\beta$ in the surroundings of motor neurons (Fig. S6). Treatment with BANA also reduced cell proliferation in the ventral horn as assessed by Ki67-positive nuclei staining (Fig. S7). In comparison, lower doses of BANA $(50 \mathrm{mg} / \mathrm{Kg} /$ day) significantly reduced microgliosis but failed to reduce astrogliosis (Fig. S8a and b). 


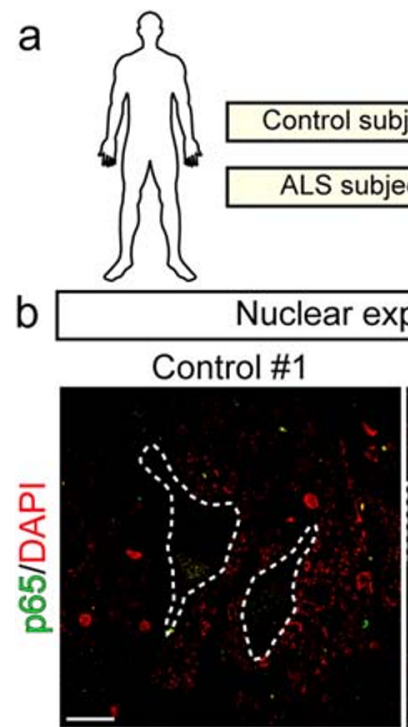

ALS \#1

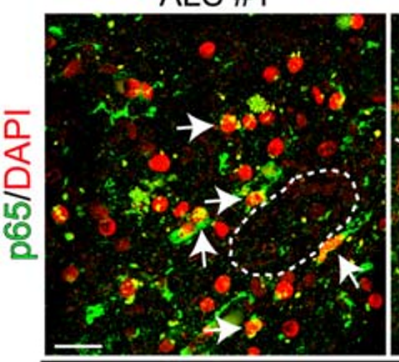

Post-mortem tissue sample collection $\longrightarrow$

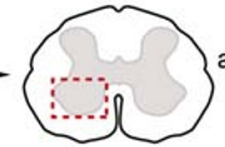
analysis of NF-kB expression in the ventral horn of the lumbar spinal cord

C

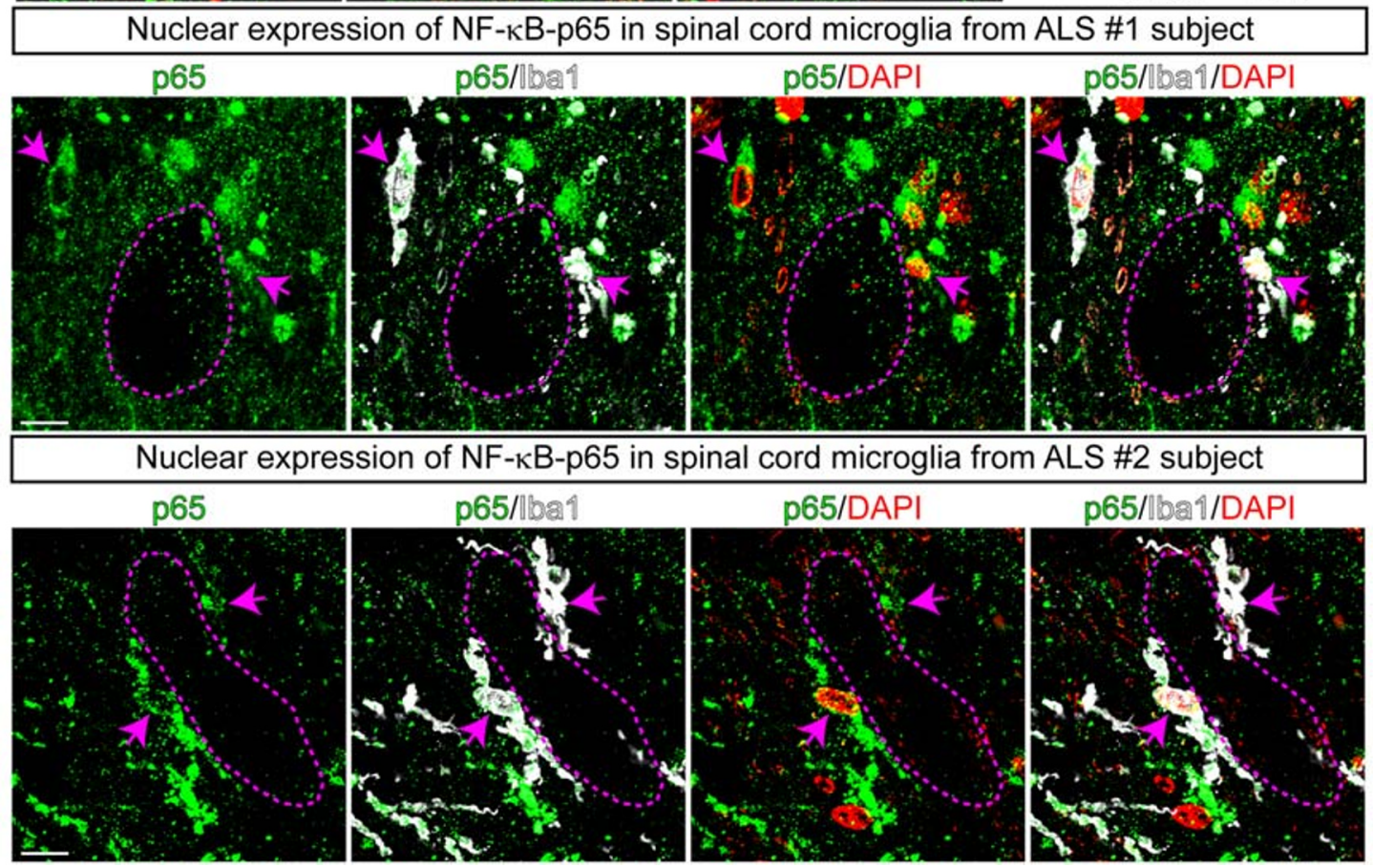

Remarkably, post-paralysis treatment with BANA at $100 \mathrm{mg}$ / $\mathrm{Kg}$ /day significantly reduced the number of Isolectin-positive microglia displaying NF-kB-p65-positive nuclei immunoreactivity, which appears typically spatially associated with motor neurons somas in the ventral horn of spinal cord of vehicle-treated
SOD $1^{\mathrm{G} 93 \mathrm{~A}}$ rats (Fig. $2 \mathrm{c}$ and $\mathrm{d}$, and Fig. S9a). Because NF- $\mathrm{BB}$ activation in microglia has been shown to induce motor neuron death in ALS models [13], the observed BANA-induced downregulation of a subpopulation of NF- $\mathrm{KB}$-positive perineuronal microglia may have therapeutic significance. 
Fig. 3 Nuclear NF-kB-p65 increased expression in microglia surrounding motor neurons in autopsied ventral spinal cords from ALS subjects. (a) Scheme of the experimental design. b) Representative confocal images showing the expression of nuclear NF-KB-p65 in the cellular microenvironment surrounding spinal motor neurons (white dotted lines) of ALS and control subjects. Upper panels: NF-kB-p65 staining (green) and DAPI (red) in control subjects. Note the lack of nuclear NF-kB-p65 expression. The panel to the right represents a higher magnification image of few Iba1-positive microglia (white) that lack cytoplasmatic or nuclear expression of NF-kB-p65 (green). Lower panels: represent confocal images showing a systematic increase expression of nuclear NF-kB-p65 in the surroundings of motor neurons in 3 sporadic ALS subjects. Arrows indicate yellow nuclei with NF-kB-p65 colocalizing with DAPI. The graph to the right shows the quantitative analysis of NF-kB-p65positive nuclei surrounding motor neurons. All quantitative data are expressed as mean \pm SEM; data were analyzed by the Mann-Whitney test, $* * * * p<0.0001 . n=3$ ALS patients and 3 controls. Scale bars $=$ $20 \mu \mathrm{m}$. (c) Higher magnification confocal images showing the cytoplasmic and nuclear expression (magenta arrows) of NF-kB-p65 in Iba1positive microglia in the ventral spinal cord of ALS subjects. Scale bars = $10 \mu \mathrm{m}$

\section{Microglia Bearing Nuclear NF-KB-p65 Surrounds Motor} Neurons in Autopsied Spinal Cords from ALS Patients To assess the potential pathogenic significance of nuclear NF-kBp65-positive microglia interacting with motor neurons in human ALS, we analyzed whether these cells also occurred in autopsied lumbar spinal cord tissue from sporadic ALS patients (Fig. 3a). As shown in Fig. 3b, there was a systematic accumulation of Iba1-/p65-positive microglia in the surroundings of spinal motor neurons in 3 ALS patients as compared

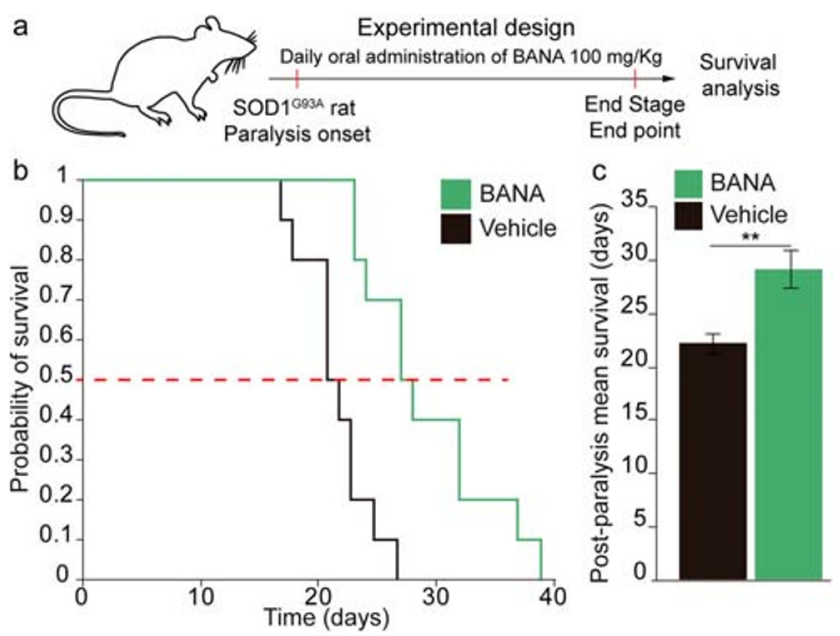

Fig. 4 BANA $(100 \mathrm{mg} / \mathrm{Kg})$ treatment after paralysis onset prolongs survival in SOD $1^{\mathrm{G} 93 \mathrm{~A}}$ rats. (a) Scheme of the experimental design. SOD $1^{\text {G93A }}$ female rats were treated with $100 \mathrm{mg} / \mathrm{Kg}$ /day of BANA or vehicle immediately after paralysis onset until the end stage $(n=10$ per group). (b) Kaplan-Meier survival curves from BANA- and vehicletreated rats. There was a statistically significant difference in the probability of survival for the BANA-treated group when compared with the vehicle, according to the Log-rank test of the Kaplan-Meier analysis $(* * * p=0.0007)$. (c) The graph shows the mean survival of both cohorts. All data are expressed as mean $\pm \mathrm{SEM}$; data were analyzed by unpaired $t$ test $* * p=0.0018$ with an almost complete absence of such phenotype in 3 control subjects. Nuclear NF-kB-p65-positive microglia were characterized by hypertrophic cell bodies, in some cases organized as cell clusters (Fig. 3c and Fig. S9b, ALS\#3 subject). This phenotype was reminiscent of senescent microglia previously reported in mutant SOD $1^{\mathrm{G} 93 \mathrm{~A}}$ rats [37]. Nuclear NF-kB-p65 was also localized in other perineuronal cells lacking Ibal staining displaying apparent astrocytic morphological features (Fig. S9).

BANA Prolongs Post-paralysis Survival in SOD $1^{\text {G93A }}$ Rats Next, we designed a randomized preclinical trial using BANA to determine how the drug affected survival in female SOD ${ }^{\text {G93A }}$ rats with hind limb onset. As reported previously [8], our rat colony develop the disease with delayed onset (187 \pm 15 days for vehicle rats), when compared with that originally described by Howland et al. [4], and the postparalysis survival in females with hind limb onset has been reproducible in nontreated animals ( $20 \pm 3.8$ days).

BANA (100 mg/kg/day) was orally administrated immediately upon abnormal gait onset and continued until the end stage (Fig. 4a). Rats treated with BANA $(n=10)$ had a $32 \%$ increase in the probability of post-paralysis survival as compared with vehicle-treated rats $(p=0.0007$ in Kaplan-Meier survival curves) (Fig. 4b). The mean post-paralysis survival time was respectively 21.8 days and 29.2 days for vehicle and BANA-treated groups, respectively (Fig. 4c).

BANA Ameliorates Motor Neuron Pathology and Neuromuscular Junction Denervation Motor neuron loss is a remarkable pathological feature in ALS patients and animal models. Therefore, we analyzed the effect of BANA on motor neuron number and size as well as on markers of neuronal damage, such as ubiquitin aggregates and nitrotyrosine staining. In vehicle-treated animals, the number of ChAT-positive motor neurons decreased by $40 \%$ with respect to nontransgenic animals in the following 15 days of disease progression after onset (Fig. 5a). In comparison, a 15-day treatment with BANA starting after paralysis onset significantly prevented subsequent motor neuron loss by $15 \%$. As compared to vehicle-treated rats, BANA also ameliorated the reduction in motor neuron size (Fig. 5a). In addition, BANA significantly prevented ubiquitin aggregation and nitrotyrosine staining in motor neurons as previously described [38, 39] (Fig. 5b). In comparison, lower doses of BANA (50 mg/Kg/day) failed to prevent motor neuron loss (Fig. S8c).

To further investigate the protective effect of BANA on motor neuron pathology in SOD $1^{\mathrm{G} 93 \mathrm{~A}}$ symptomatic rats, we assessed the number of synaptic terminals contacting motor neuron cell bodies in the ventral spinal cord as well as the denervation of neuromuscular junctions. Postparalysis treatment with $100 \mathrm{mg} / \mathrm{Kg} /$ day of BANA 
a

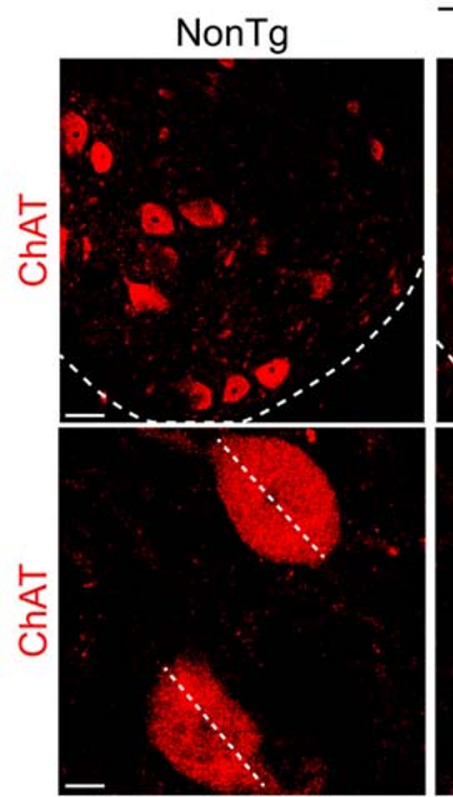

SOD1 ${ }^{\text {G93A }}$ advanced paralysis
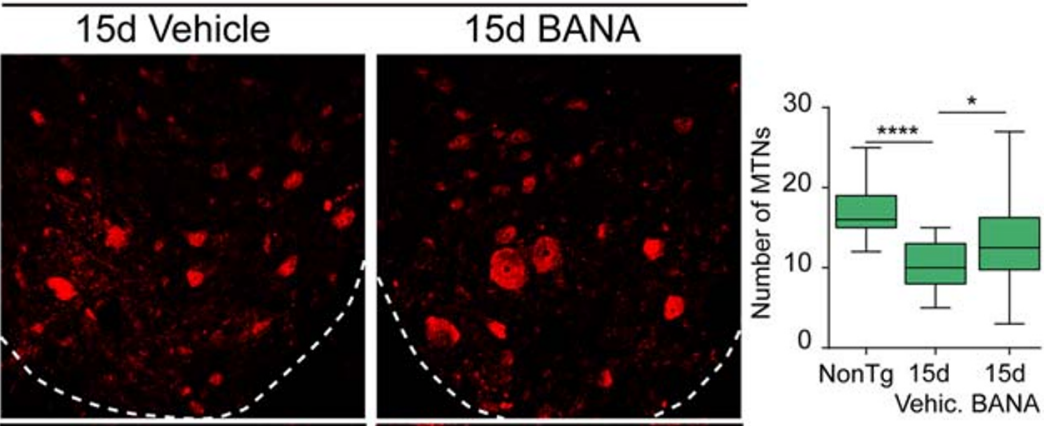

b
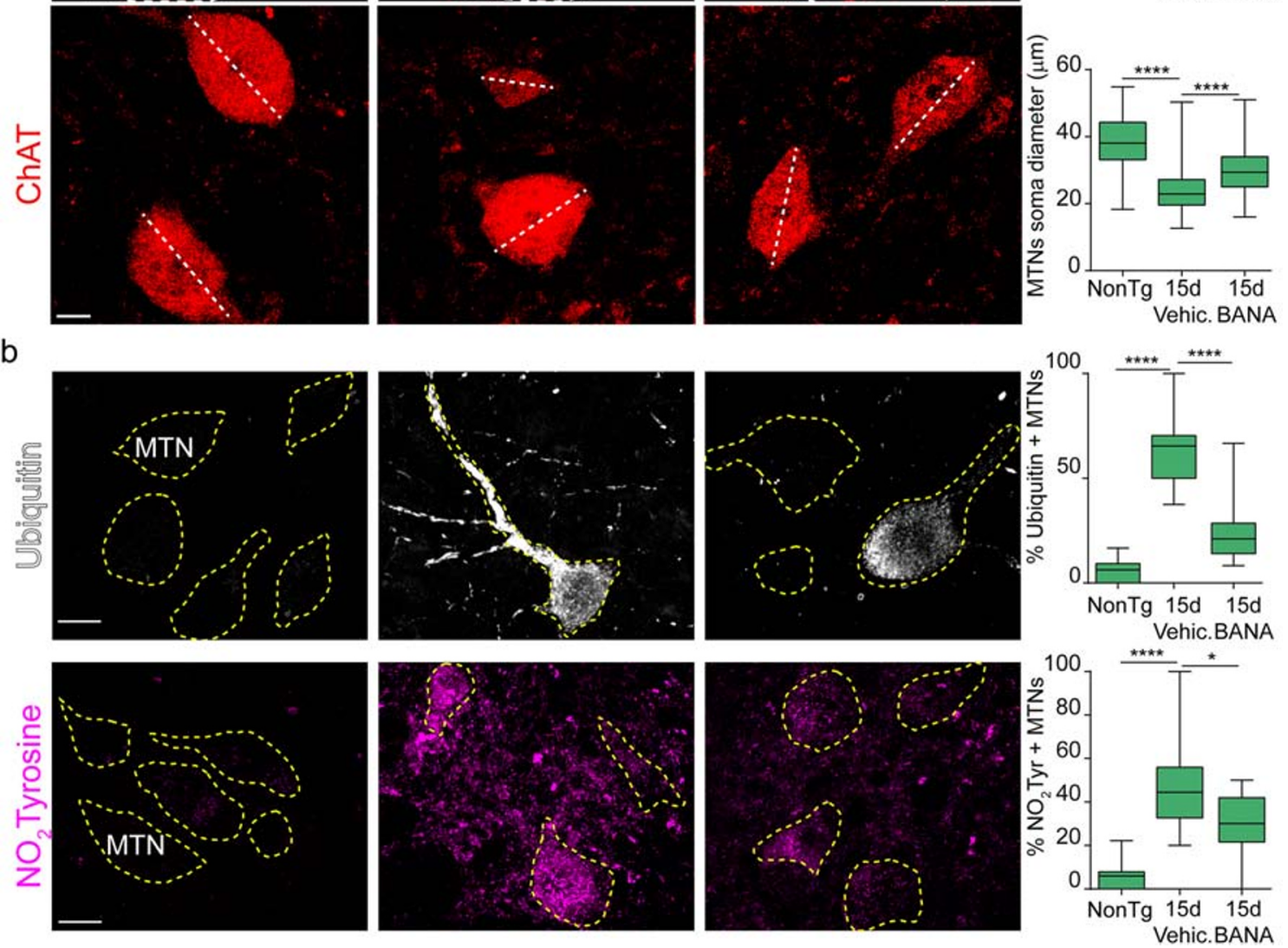

Fig. 5 BANA (100 mg/Kg) ameliorates motor neuron pathology. (a) Upper panels: confocal images of ChAT-positive motor neurons in the lumbar spinal cord (dotted line indicates the limit between white and gray matter) of nontransgenic, vehicle-treated, and BANA-treated SOD1 $1{ }^{\mathrm{G} 93 \mathrm{~A}}$ rats immediately after the first signs of paralysis onset during 15 days. The graph represents the quantitative analysis of the number of motor neurons in laminae VII and IX of the ventral horn among conditions. All data are expressed as mean \pm SEM; data were analyzed by the KruskalWallis test followed by Dunn's multiple comparisons test, ${ }^{*} p=0.0217$ $* * * * p<0.0001 . n=4$ animals per condition. Scale bar $=50 \mu \mathrm{m}$. Lower panels: represent the immunohistochemical analysis of motor neuron soma size. The graph represents the quantitation of the motor neuron soma diameter in the different experimental conditions. All data are expressed as mean \pm SEM; data were analyzed by the Kruskal-Wallis test followed by Dunn's multiple comparisons test, $* * * * p<0.0001 . n=4$ animals per condition. Scale bar $=20 \mu \mathrm{m}$. (b) Immunohistochemical analysis of ubiquitin and nitrotyrosine $\left(\mathrm{NO}_{2}\right.$ Tyrosine) aggregation in motor neurons (yellow dotted lines) of the lumbar spinal cord. Vehicle-treated rats showed a significantly increased number of ubiquitinated motor neurons and increased expression of $\mathrm{NO}_{2}$ Tyrosine when compared with nontransgenic littermates. Post-paralysis treatment with BANA significantly prevented ubiquitin aggregation and $\mathrm{NO}_{2}$ Tyrosine accumulation in surviving motor neurons. The graph to the right shows the number of motor neurons showing ubiquitin and $\mathrm{NO}_{2}$ Tyrosine aggregates in the ventral horn of the spinal cord. Data are expressed as mean $\pm \mathrm{SEM}$; data were analyzed by the Kruskal-Wallis test followed by Dunn's multiple comparisons test, $* p=0.0219 * * * * p<0.0001 . n=4$ animals per condition. Scale bars $=20 \mu \mathrm{m}$ significantly prevented the loss of VGlut-1-positive and synaptophysin-positive synaptic terminals as compared with vehicle-treated rats (Fig. 6). In accordance, BANA treatment significantly reduced motor endplate denervation in the EDL muscle as compared to vehicle-treated rats (Fig. 6). 
a
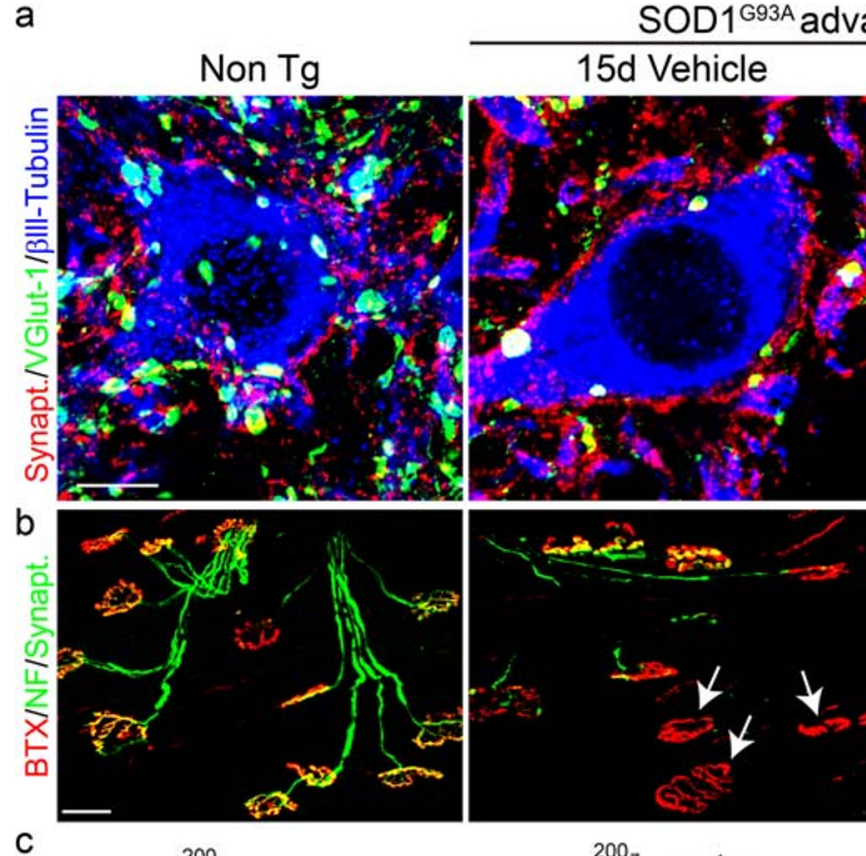

15d BANA
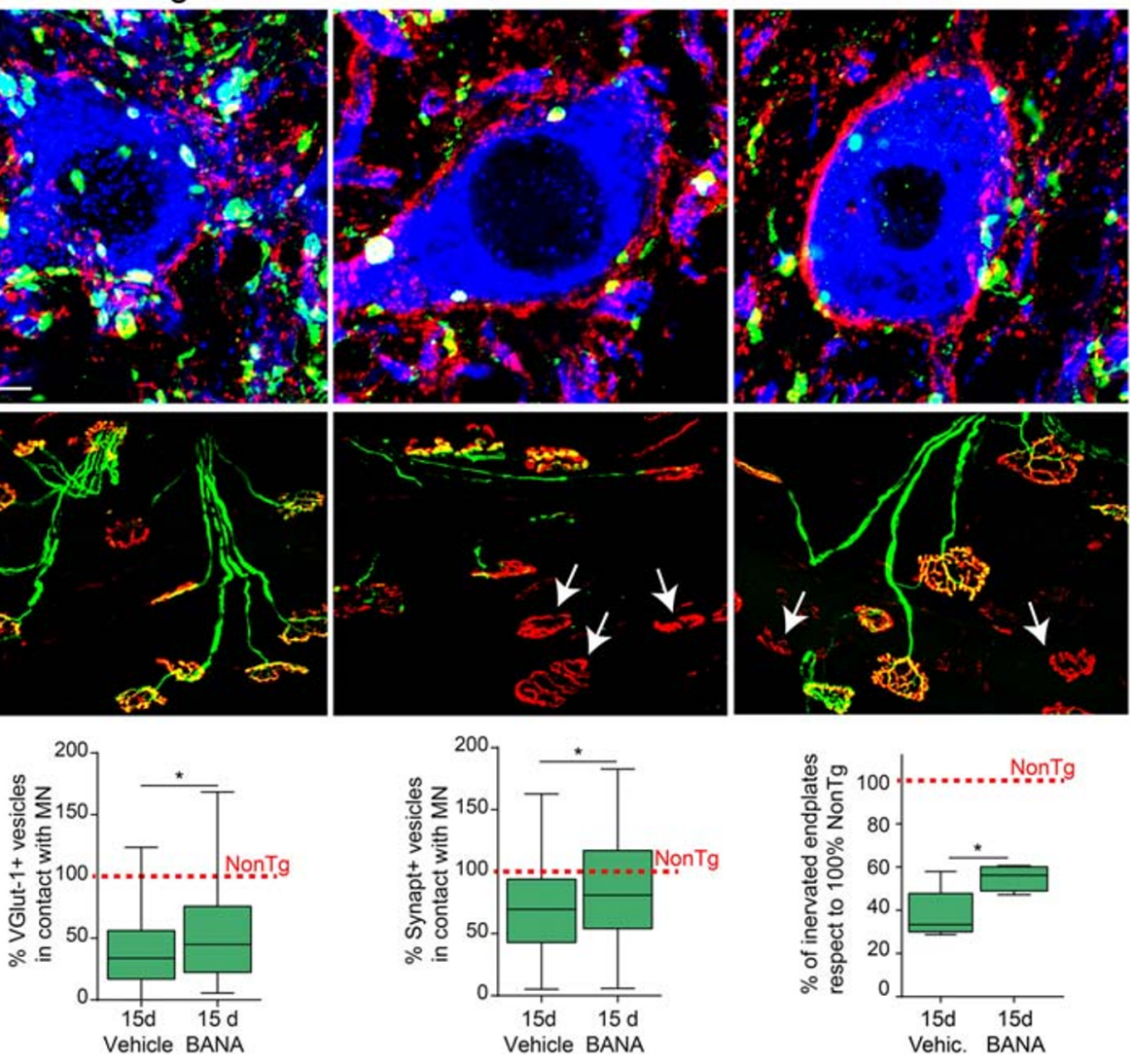

Fig. 6 BANA (100 mg/Kg) reduces synaptic input loss of motor neurons and prevents NMJ denervation. (a) Representative confocal images show-

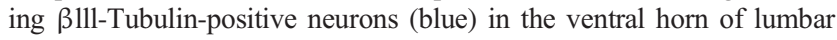
spinal cord costained with synaptic vesicle markers VGlut-1 (green) and synaptophysin (red). Note that post-paralysis treatment with BANA after $15 \mathrm{~d}$ of from onset significantly prevented synaptic terminal loss that contact motor neuron cell bodies. Scale bar $=10 \mu \mathrm{m}$. (b) NMJs denervation analysis in whole-mounted EDL muscles. The panels show representative confocal images used to assess the innervation pattern of NMJs in different experimental conditions. $\alpha$-bungarotoxin-FITC (red) staining was used to analyze motor endplates. Synaptophysin-AlexFluor 555

and heavy chain of neurofilaments-Alexa Fluor 555 (green) were used to visualize the motor axon branches and presynaptic terminals. Arrows indicate typical denervated motor endplates. Scale bar $=50 \mu \mathrm{m}$. (c) Graphs represent the quantitative analysis of synaptic vesicles in contact with motor neuron cell bodies (left and middle graphs), and NMJ occupancy defined as the overlapping of neurofilament/synaptophysin and $\alpha$ BTX staining (graph to the right), expressed as percent with respect to the nontransgenic condition. All data are expressed as mean $\pm \mathrm{SEM}$; data were analyzed by the Mann-Whitney comparisons test or unpaired $t$ test between vehicle and BANA groups, ${ }^{*} p=0.0474$ (VGlut-1), ${ }^{*} p=0.0447$ (synaptophysin) $* p=0.0324$ (NMJ). $n=4$ animals per condition

\section{Discussion}

Anti-inflammatory benzoic acid derivatives, including salicylic and acetylsalicylic acids as well as vanillic acid, are recognized to inhibit NF- $\mathrm{KB}[27,40]$, but they are poorly effective in therapeutically controlling deleterious microglial activation. Here, we show evidence that the nitroalkene benzoic acid derivative BANA, modulates the neuroinflammation associated with the ALS progression in $\mathrm{SOD} 1^{\mathrm{G} 93 \mathrm{~A}}$ rats. BANA reduced microgliosis and prevented the emergence of microglia displaying nuclear NF-KB-p65 typically surrounding motor neurons in the degenerating spinal cord of both SOD $1^{\mathrm{G} 93 \mathrm{~A}}$ rats and ALS patients, further indicating the potential relevance of this subset of neurotoxic microglia as a target of BANA. Strikingly, the treatment of already

paralytic rats with BANA resulted in a $32 \%$ increase in postparalysis survival. Therefore, our data extend previous studies showing that genetic or pharmacological inhibition of NF-KB pathways in ALS models can reduce disease symptoms and prolong survival $[13,16,41]$, and for the first time we report the benzoic acid derivative BANA as an NF- $\mathrm{KB}$ inhibitor that exerts a protective effect when delivered post-paralysis in an ALS model. Such a therapeutic approach seems adapted in the clinical setting of ALS where drug treatment is initiated only after the development of motor symptoms. Translated to ALS patients, the protective effect of BANA might result in delayed paralysis progression and extended survival.

BANA was particularly active to target NF- $\mathrm{KB}$ in microglia cultures including those isolated from the symptomatic SOD $1^{\mathrm{G} 93 \mathrm{~A}}$ rat spinal cord. SOD $1^{\mathrm{G} 93 \mathrm{~A}}$ microglia can exhibit 
an aberrant phenotype with increased proliferation and neurotoxic potential $[5,6]$, suggesting they fuel local inflammation and exert a deleterious influence on neuronal survival. In ALS models, the classical NF- $\mathrm{kB}$ pathway is related to persistent microglial activation with accelerated disease progression [13, 42]. Here, we identified a specific subset of microglia displaying NF-KB-p65 nuclear translocation localized in the close surrounding of spinal motor neurons. Such microglia appeared to functionally interact with motor neurons in autopsied spinal cords from sporadic ALS patients and also in symptomatic $\mathrm{SOD} 1{ }^{\mathrm{G} 93 \mathrm{~A}}$ rats, but not in respective controls. These results are in accordance with previous reports showing $20 \%$ of spinal cord microglia displaying nuclear NF-KB-p65 colocalized with TDP43 in sporadic ALS subjects [16]. Our results strongly suggest a clear causal association of nuclear $\mathrm{NF}-\mathrm{KB}$ in microglia with progressive motor neuron damage and neuromuscular junction loss. Notably, post-paralysis treatment with BANA for 15 days resulted in a significant reduction of perineuronal microglia bearing NF-kB-p65positive nuclei, suggesting the potential of BANA to target this particular subset of microglia.

$\mathrm{SOD} 1^{\mathrm{G} 93 \mathrm{~A}}$ rats receiving post-paralysis treatment with BANA not only had prolonged survival and decreased nuclear NF-KB-p65-positive microglia but also exhibited healthier motor neurons as assessed by reduction of neurons displaying nitrotyrosine and ubiquitin staining. Accumulation of ubiquitin immunoreactivity denotes ER stress and proteinopathy $[43,44]$ while nitrotyrosine staining is a marker of oxidative and nitrative stress preceding apoptosis in motor neurons [39, 45]. BANA treatment also preserved the reduction of the number, size, and synaptic inputs of spinal motor neurons as well as the number of innervated neuromuscular junctions in the EDL muscle, further confirming the neuroprotective effect of BANA in ALS SOD1 ${ }^{\mathrm{G} 93 \mathrm{~A}}$ rats.

Because of the nitroalkene functional electrophilic group, BANA appears as a covalent binder drug with the ability to react with nucleophiles such as glutathione or sulfhydryl groups in cysteine residues. The advantages and limitations of such a drug class have been previously reviewed [46, 47]. However, the reactivity with cysteine residues in key protein complexes controlling inflammatory signaling pathways such as NF-KB, Keap1-Nrf2, and PPAR $\gamma$ may be of significant therapeutic value in neurodegenerative diseases [48-51]. In this context, BANA may be compared with electrophilic endogenous nitro-fatty acids that in addition to inhibiting NF- $\mathrm{KB}$ by covalently targeting NF-KB-p65, also induce the Keap1/ Nrf2 pathways and exert neuroprotective effects in ALS models [20]. BANA could also be compared with the electrophilic drug dimethyl fumarate approved for the treatment of multiple sclerosis and currently assayed in ALS clinical trials $[35,52,53]$. While the mechanism of action of dimethyl fumarate seems to primarily involve Keap1/Nrf2 activation and NF-KB inhibition [54, 55], in our experimental setup it failed to inhibit NF-KB stimulated by LPS and TNF $\alpha$ in cultured microglia, suggesting a different mechanism of action than BANA.

It is unlikely that BANA's neuroprotective effect in SOD $1{ }^{\mathrm{G} 93 \mathrm{~A}}$ rats is solely due to its specific effect on NF-KBactivated microglia. Firstly, targeting NF-kB is complicated due to its ubiquitous expression and diverse functions in different cell types [12]. Also, BANA could target other cytoprotective signaling pathways in addition to NF- $\mathrm{KB}$, including Nrf2-ARE and/or PPAR $\gamma$ as is the case of other electrophilic nitroalkene-containing compounds [19, 22]. BANA could also target neural and immune cells in the peripheral nervous system, which are also known to modulate paralysis progression in ALS [33, 56, 57]. Finally, BANA may also target relevant cysteine residues that affect mutant SOD1 conformational states and neurotoxicity, thus comparing with the proposed mechanisms of action of CuATSM [58, 59].

After oral administration, BANA appears to reach a low micromolar concentration in plasma. Although we have not directly determined the uptake of BANA through the bloodspinal cord barrier, it can be inferred that BANA can be a substrate of monocarboxylic acid type- 1 transporters, which are known to transport benzoic acid and salicylic acid to the CNS [60]. Evidence also indicates significant alterations in the blood-spinal cord barrier in ALS, including endothelial cells and pericyte degeneration, capillary leakage, downregulation of tight junction proteins, and microhemorrhages [61], which likely play a pathogenic mechanism aggravating motor neuron damage [62]. Because restoring blood-brain-barrier integrity retards the disease process in ALS animal models [62], BANA might also protect vascular pathology in ALS through modulation of NF- $\mathrm{KB}$ signaling in endothelial cells and pericytes [63].

In conclusion, here we show evidence for the neuroprotective effect of the nitroalkene benzoic acid derivative BANA in a model of inherited ALS exerting a diseasemodifying effect when administered after paralysis onset. While the inhibitory effects of BANA on NF- KB activation are systemic and possibly involves multifaceted cell types, strikingly, the present data identifies a subset of NF-kB-positive ALS-associated microglia surrounding spinal motor neurons as pathogenic-relevant targets of the drug. Here we show for the first time the emergence of such perineuronal NF-kB-positive microglia in autopsy samples from sporadic ALS cases, providing further insight on pathological microglia-motor neuron crosstalk in ALS that can be pharmacology targeted by BANA.

Acknowledgments We want to thank Tabaré de los Campos from the Microscopy Unit at Institut Pasteur de Montevideo for his assistance in imaging analysis. We also want to thank the staff from the Transgenic and Experimental Animal Unit from Institut Pasteur de Montevideo. We are grateful to our ALS patients who donated their skeletal muscle tissues postmortem to advance ALS research. 
Required Author Forms Disclosure forms provided by the authors are available with the online version of this article.

Author Contributions SI, CE, CB, LB, and ET designed the study. SI, MK, VV, JRD, MI, PI, WP, GVL, APA, KP, MBF, PK, YS, YK, and ET prepared samples and performed experiments. SI, MI, JRD, KP, LB, and ET analyzed data. SI, LB, and ET prepared the manuscript. All authors contributed to the discussion of the results and edited and approved the final version of the manuscript.

Funding This work was supported by Institut Pasteur de Montevideo FOCEM Mercosur (COF 03/11), Agencia Nacional de Investigación e Innovación (ANII) (I+I Proyecto), Programa de Desarrollo de las Ciencias Básicas (PEDECIBA), Sistema Nacional de Investigadores (SNI), EOLO PHARMA S.A and affiliates. This work was also supported by the National Institute of Neurological Disorders and Stroke, R01NS092651 and R21NS111275-01 (PHK), and by the Dept. of Veterans Affairs, BX001148 (PHK).

\section{Compliance with Ethical Standards}

Conflict of Interest $\mathrm{MI}$ is supported by EOLO PHARMA. GVL, CE, and $\mathrm{CB}$ hold shares in EOLO PHARMA S.A. and affiliates.

\section{References}

1. Brown RH, Al-Chalabi A. Amyotrophic Lateral Sclerosis. N Engl J Med 2017;377(2):162-72. https://doi.org/10.1056/ NEJMra1603471.

2. Miller RG, Mitchell JD, Lyon M, Moore DH. Riluzole for amyotrophic lateral sclerosis (ALS)/motor neuron disease (MND). Amyotroph Lateral Scler Other Motor Neuron Disord 2003;4(3): 191-206.

3. Writing G, Edaravone ALSSG. Safety and efficacy of edaravone in well defined patients with amyotrophic lateral sclerosis: a randomised, double-blind, placebo-controlled trial. Lancet Neurol 2017;16(7):505-12. https://doi.org/10.1016/S1474-4422(17) 30115-1.

4. Howland DS, Liu J, She Y, Goad B, Maragakis NJ, Kim B, et al. Focal loss of the glutamate transporter EAAT2 in a transgenic rat model of SOD1 mutant-mediated amyotrophic lateral sclerosis (ALS). Proc Natl Acad Sci U S A. 2002;99(3):1604-9. https://doi. org/10.1073/pnas.032539299.

5. Diaz-Amarilla P, Olivera-Bravo S, Trias E, Cragnolini A, MartinezPalma L, Cassina P, et al. Phenotypically aberrant astrocytes that promote motoneuron damage in a model of inherited amyotrophic lateral sclerosis. Proc Natl Acad Sci U S A. 2011;108(44):1812631. https://doi.org/10.1073/pnas.1110689108.

6. Trias E, Diaz-Amarilla P, Olivera-Bravo S, Isasi E, Drechsel DA, Lopez N, et al. Phenotypic transition of microglia into astrocyte-like cells associated with disease onset in a model of inherited ALS. Front Cell Neurosci 2013;7:274. https://doi.org/10.3389/fncel. 2013.00274

7. Trias E, Ibarburu S, Barreto-Nunez R, Barbeito L. Significance of aberrant glial cell phenotypes in pathophysiology of amyotrophic lateral sclerosis. Neurosci Lett 2017;636:27-31. https://doi.org/10. 1016/j.neulet.2016.07.052.

8. Trias E, Ibarburu S, Barreto-Nunez R, Babdor J, Maciel TT, Guillo $\mathrm{M}$, et al. Post-paralysis tyrosine kinase inhibition with masitinib abrogates neuroinflammation and slows disease progression in inherited amyotrophic lateral sclerosis. J Neuroinflammation 2016;13(1):177. https://doi.org/10.1186/s12974-016-0620-9.
9. Mora JS, Genge A, Chio A, Estol CJ, Chaverri D, Hernandez M, et al. Masitinib as an add-on therapy to riluzole in patients with amyotrophic lateral sclerosis: a randomized clinical trial. Amyotroph Lateral Scler Frontotemporal Degener 2020;21(1-2): 5-14. https://doi.org/10.1080/21678421.2019.1632346.

10. Tak PP, Firestein GS. NF-kappaB: a key role in inflammatory diseases. J Clin Invest 2001;107(1):7-11. https://doi.org/10.1172/ JCI1 1830 .

11. Kaltschmidt B, Kaltschmidt C. NF-kappaB in the nervous system. Cold Spring Harb Perspect Biol 2009;1(3):a001271. https://doi.org/ 10.1101/cshperspect.a001271.

12. Liu T, Zhang L, Joo D, Sun SC. NF-kappaB signaling in inflammation. Signal Transduct Targete Therapy 2017;2. https://doi.org/ 10.1038/sigtrans.2017.23.

13. Frakes AE, Ferraiuolo L, Haidet-Phillips AM, Schmelzer L, Braun L, Miranda CJ, et al. Microglia induce motor neuron death via the classical NF-kappaB pathway in amyotrophic lateral sclerosis. Neuron. 2014;81(5):1009-23. https://doi.org/10.1016/j.neuron. 2014.01.013.

14. Dresselhaus EC, Meffert MK. Cellular Specificity of NF-kappaB Function in the Nervous System. Front Immunol 2019;10:1043. https://doi.org/10.3389/fimmu.2019.01043.

15. Ju Hwang C, Choi DY, Park MH, Hong JT. NF-kappaB as a Key Mediator of Brain Inflammation in Alzheimer's Disease. CNS Neurol Disord Drug Targets 2019;18(1):3-10. https://doi.org/10. 2174/1871527316666170807130011.

16. Swarup V, Phaneuf D, Dupre N, Petri S, Strong M, Kriz J, et al. Deregulation of TDP-43 in amyotrophic lateral sclerosis triggers nuclear factor kappaB-mediated pathogenic pathways. J Exp Med. 2011;208(12):2429-47. https://doi.org/10.1084/jem.20111313.

17. Sako W, Ito H, Yoshida M, Koizumi H, Kamada M, Fujita K, et al. Nuclear factor kappa B expression in patients with sporadic amyotrophic lateral sclerosis and hereditary amyotrophic lateral sclerosis with optineurin mutations. Clin Neuropathol 2012;31(6):418-23. https://doi.org/10.5414/NP300493.

18. Khoo NKH, Li L, Salvatore SR, Schopfer FJ, Freeman BA. Electrophilic fatty acid nitroalkenes regulate Nrf2 and NF-kappaB signaling:A medicinal chemistry investigation of structure-function relationships. Sci Rep 2018;8(1):2295. https://doi.org/10.1038/ s41598-018-20460-8.

19. Cui T, Schopfer FJ, Zhang J, Chen K, Ichikawa T, Baker PR, et al. Nitrated fatty acids: Endogenous anti-inflammatory signaling mediators. J Biol Chem 2006;281(47):35686-98. https://doi.org/10. 1074/jbc.M603357200.

20. Diaz-Amarilla P, Miquel E, Trostchansky A, Trias E, Ferreira AM, Freeman BA, et al. Electrophilic nitro-fatty acids prevent astrocytemediated toxicity to motor neurons in a cell model of familial amyotrophic lateral sclerosis via nuclear factor erythroid 2-related factor activation. Free Radic Biol Med 2016;95:112-20. https://doi.org/ 10.1016/j.freeradbiomed.2016.03.013.

21. Rodriguez-Duarte J, Dapueto R, Galliussi G, Turell L, Kamaid A, Khoo NKH, et al. Electrophilic nitroalkene-tocopherol derivatives: synthesis, physicochemical characterization and evaluation of antiinflammatory signaling responses. Sci Rep 2018;8(1):12784. https://doi.org/10.1038/s41598-018-31218-7.

22. Rodriguez-Duarte J, Galliussi G, Dapueto R, Rossello J, Malacrida L, Kamaid A, et al. A novel nitroalkene-alpha-tocopherol analogue inhibits inflammation and ameliorates atherosclerosis in Apo E knockout mice. Br J Pharmacol 2019;176(6):757-72. https://doi. org/10.1111/bph.14561.

23. Bridges JW, French MR, Smith RL, Williams RT. The fate of benzoic acid in various species. Biochem J 1970;118(1):47-51. https://doi.org/10.1042/bj1180047.

24. Leonard JV, Morris AA. Urea cycle disorders. Semin Neonatol 2002;7(1):27-35. https://doi.org/10.1053/siny.2001.0085. 
25. Modi KK, Roy A, Brahmachari S, Rangasamy SB, Pahan K. Cinnamon and Its Metabolite Sodium Benzoate Attenuate the Activation of p21rac and Protect Memory and Learning in an Animal Model of Alzheimer's Disease. PLoS One 2015;10(6): e0130398. https://doi.org/10.1371/journal.pone.0130398.

26. Khasnavis S, Pahan K. Sodium benzoate, a metabolite of cinnamon and a food additive, upregulates neuroprotective Parkinson disease protein DJ-1 in astrocytes and neurons. J Neuroimmune Pharmacol 2012;7(2):424-35. https://doi.org/10.1007/s11481-011-9286-3.

27. Yin MJ, Yamamoto Y, Gaynor RB. The anti-inflammatory agents aspirin and salicylate inhibit the activity of I(kappa)B kinase-beta. Nature. 1998;396(6706):77-80. https://doi.org/10.1038/23948.

28. Grilli M, Pizzi M, Memo M, Spano P. Neuroprotection by aspirin and sodium salicylate through blockade of NF-kappaB activation. Science. 1996;274(5291):1383-5. https://doi.org/10.1126/science. 274.5291.1383.

29. Albrecht P, Bouchachia I, Goebels N, Henke N, Hofstetter HH, Issberner A, et al. Effects of dimethyl fumarate on neuroprotection and immunomodulation. J Neuroinflammation 2012;9:163. https:// doi.org/10.1186/1742-2094-9-163.

30. Miller SA, White JA, Chowdhury R, Gales DN, Tameru B, Tiwari $\mathrm{AK}$, et al. Effects of consumption of whole grape powder on basal NF-kappaB signaling and inflammatory cytokine secretion in a mouse model of inflammation. J Nutr Intermed Metabol 2018;11: 1-8. https://doi.org/10.1016/j.jnim.2017.11.002.

31. Mastropietro G, Tiscornia I, Perelmuter K, Astrada S, BollatiFogolin M. HT-29 and Caco-2 reporter cell lines for functional studies of nuclear factor kappa B activation. Mediat Inflamm 2015;2015:860534. https://doi.org/10.1155/2015/860534.

32. Thomsen GM, Gowing G, Latter J, Chen M, Vit JP, Staggenborg $\mathrm{K}$, et al. Delayed disease onset and extended survival in the SOD1G93A rat model of amyotrophic lateral sclerosis after suppression of mutant SOD1 in the motor cortex. J Neurosci. 2014;34(47):15587-600. https://doi.org/10.1523/JNEUROSCI. 2037-14.2014.

33. Trias E, Ibarburu S, Barreto-Nunez R, Varela V, Moura IC, Dubreuil $\mathrm{P}$, et al. Evidence for mast cells contributing to neuromuscular pathology in an inherited model of ALS. JCI Insight 2017;2(20). https://doi.org/10.1172/jci.insight.95934.

34. Kovacs M, Trias E, Varela V, Ibarburu S, Beckman JS, Moura IC, et al. CD34 Identifies a Subset of Proliferating Microglial Cells Associated with Degenerating Motor Neurons in ALS. Int J Mol Sci 2019;20(16). https://doi.org/10.3390/ijms20163880.

35. Vucic S, Ryder J, Mekhael L, Rd H, Mathers S, Needham M, et al. Phase 2 randomized placebo controlled double blind study to assess the efficacy and safety of tecfidera in patients with amyotrophic lateral sclerosis (TEALS Study): Study protocol clinical trial (SPIRIT Compliant). Medicine. 2020;99(6):e18904. https://doi. org/10.1097/MD.0000000000018904.

36. Brahmachari S, Jana A, Pahan K. Sodium benzoate, a metabolite of cinnamon and a food additive, reduces microglial and astroglial inflammatory responses. J Immunol 2009;183(9):5917-27. https:// doi.org/10.4049/jimmunol.0803336.

37. Trias E, Beilby PR, Kovacs M, Ibarburu S, Varela V, BarretoNunez R, et al. Emergence of Microglia Bearing Senescence Markers During Paralysis Progression in a Rat Model of Inherited ALS. Front Aging Neurosci 2019;11:42. https://doi.org/10.3389/ fnagi.2019.00042.

38. Leigh PN, Whitwell H, Garofalo O, Buller J, Swash M, Martin JE, et al. Ubiquitin-immunoreactive intraneuronal inclusions in amyotrophic lateral sclerosis. Morphology, distribution, and specificity. Brain J Neurol 1991;114 ( Pt 2):775-88.

39. Beal MF, Ferrante RJ, Browne SE, Matthews RT, Kowall NW, Brown RH, Jr. Increased 3-nitrotyrosine in both sporadic and familial amyotrophic lateral sclerosis. Ann Neurol 1997;42(4):64454. https://doi.org/10.1002/ana.410420416.
40. Kim MC, Kim SJ, Kim DS, Jeon YD, Park SJ, Lee HS, et al. Vanillic acid inhibits inflammatory mediators by suppressing NFkappaB in lipopolysaccharide-stimulated mouse peritoneal macrophages. Immunopharmacol Immunotoxicol 2011;33(3):525-32. https://doi.org/10.3109/08923973.2010.547500.

41. Patel P, Julien JP, Kriz J. Early-stage treatment with Withaferin A reduces levels of misfolded superoxide dismutase 1 and extends lifespan in a mouse model of amyotrophic lateral sclerosis. Neurotherapeutics 2015;12(1):217-33. https://doi.org/10.1007/ s13311-014-0311-0.

42. McCauley ME, Baloh RH. Inflammation in ALS/FTD pathogenesis. Acta Neuropathol 2019;137(5):715-30. https://doi.org/10.1007/ s00401-018-1933-9.

43. Shruthi K, Reddy SS, Chitra PS, Reddy GB. Ubiquitin-proteasome system and ER stress in the brain of diabetic rats. J Cell Biochem 2019;120(4):5962-73. https://doi.org/10.1002/jcb.27884.

44. Dantuma NP, Bott LC. The ubiquitin-proteasome system in neurodegenerative diseases: precipitating factor, yet part of the solution. Front Mol Neurosci 2014;7:70. https://doi.org/10.3389/fnmol. 2014.00070.

45. Estevez AG, Spear N, Manuel SM, Radi R, Henderson CE, Barbeito L, et al. Nitric oxide and superoxide contribute to motor neuron apoptosis induced by trophic factor deprivation. J Neurosci 1998;18(3):923-31.

46. Lipton SA, Rezaie T, Nutter A, Lopez KM, Parker J, Kosaka K, et al. Therapeutic advantage of pro-electrophilic drugs to activate the Nrf2/ARE pathway in Alzheimer's disease models. Cell Death Dis 2016;7(12):e2499. https://doi.org/10.1038/cddis.2016.389.

47. Schopfer FJ, Vitturi DA, Jorkasky DK, Freeman BA. Nitro-fatty acids: New drug candidates for chronic inflammatory and fibrotic diseases. Nitric Oxide Biol Chem 2018;79:31-7. https://doi.org/10. 1016/j.niox.2018.06.006.

48. Bright JJ, Kanakasabai S, Chearwae W, Chakraborty S. PPAR Regulation of Inflammatory Signaling in CNS Diseases. PPAR Res 2008;2008:658520. https://doi.org/10.1155/2008/658520.

49. Corona JC, Duchen MR. PPARgamma as a therapeutic target to rescue mitochondrial function in neurological disease. Free Radic Biol Med 2016;100:153-63. https://doi.org/10.1016/j. freeradbiomed.2016.06.023.

50. Satoh T, Okamoto SI, Cui J, Watanabe Y, Furuta K, Suzuki M, et al. Activation of the Keap1/Nrf2 pathway for neuroprotection by electrophilic [correction of electrophillic] phase II inducers. Proc Natl Acad Sci U S A. 2006;103(3):768-73. https://doi.org/10. 1073/pnas.0505723102.

51. Srinivasan M, Lahiri DK. Significance of NF-kappaB as a pivotal therapeutic target in the neurodegenerative pathologies of Alzheimer's disease and multiple sclerosis. Expert Opin Ther Targets 2015;19(4):471-87. https://doi.org/10.1517/14728222. 2014.989834.

52. Bomprezzi R. Dimethyl fumarate in the treatment of relapsingremitting multiple sclerosis: an overview. Ther Adv Neurol Disord 2015;8(1):20-30. https://doi.org/10.1177/ 1756285614564152 .

53. Carlstrom KE, Ewing E, Granqvist M, Gyllenberg A, Aeinehband $\mathrm{S}$, Enoksson SL, et al. Therapeutic efficacy of dimethyl fumarate in relapsing-remitting multiple sclerosis associates with ROS pathway in monocytes. Nat Commun 2019;10(1):3081. https://doi.org/10. 1038/s41467-019-11139-3.

54. Mills EA, Ogrodnik MA, Plave A, Mao-Draayer Y. Emerging Understanding of the Mechanism of Action for Dimethyl Fumarate in the Treatment of Multiple Sclerosis. Front Neurol 2018;9:5. https://doi.org/10.3389/fneur.2018.00005.

55. Kastrati I, Siklos MI, Calderon-Gierszal EL, El-Shennawy L, Georgieva G, Thayer EN, et al. Dimethyl Fumarate Inhibits the Nuclear Factor kappaB Pathway in Breast Cancer Cells by 
Covalent Modification of p65 Protein. J Biol Chem. 2016;291(7): 3639-47. https://doi.org/10.1074/jbc.M115.679704.

56. Trias E, King PH, Si Y, Kwon Y, Varela V, Ibarburu S, et al. Mast cells and neutrophils mediate peripheral motor pathway degeneration in ALS. JCI Insight 2018;3(19). https://doi.org/10.1172/jci. insight.123249.

57. Graves MC, Fiala M, Dinglasan LA, Liu NQ, Sayre J, Chiappelli F, et al. Inflammation in amyotrophic lateral sclerosis spinal cord and brain is mediated by activated macrophages, mast cells and T cells. Amyotroph Lateral Scler Other Motor Neuron Disord. 2004;5(4): 213-9. https://doi.org/10.1080/14660820410020286.

58. Nagano S, Takahashi Y, Yamamoto K, Masutani H, Fujiwara N, Urushitani $\mathrm{M}$, et al. A cysteine residue affects the conformational state and neuronal toxicity of mutant SOD1 in mice: relevance to the pathogenesis of ALS. Hum Mol Genet 2015;24(12):3427-39. https://doi.org/10.1093/hmg/ddv093.

59. Williams JR, Trias E, Beilby PR, Lopez NI, Labut EM, Bradford CS, et al. Copper delivery to the CNS by CuATSM effectively treats motor neuron disease in $\operatorname{SOD}(\mathrm{G} 93 \mathrm{~A})$ mice co-expressing the Copper-Chaperone-for-SOD. Neurobiol Dis 2016;89:1-9. https://doi.org/10.1016/j.nbd.2016.01.020.
60. Tsuji A. Small molecular drug transfer across the blood-brain barrier via carrier-mediated transport systems. NeuroRx 2005;2(1):5462. https://doi.org/10.1602/neurorx.2.1.54.

61. Garbuzova-Davis S, Sanberg PR. Blood-CNS Barrier Impairment in ALS patients versus an animal model. Front Cell Neurosci 2014;8:21. https://doi.org/10.3389/fncel.2014.00021.

62. Winkler EA, Sengillo JD, Sagare AP, Zhao Z, Ma Q, Zuniga E, et al. Blood-spinal cord barrier disruption contributes to early motor-neuron degeneration in ALS-model mice. Proc Natl Acad Sci U S A. 2014;111(11):E1035-42. https://doi.org/10.1073/pnas. 1401595111.

63. Jansson D, Rustenhoven J, Feng S, Hurley D, Oldfield RL, Bergin PS, et al. A role for human brain pericytes in neuroinflammation. J Neuroinflammation 2014;11:104. https://doi.org/10.1186/17422094-11-104.

Publisher's Note Springer Nature remains neutral with regard to jurisdictional claims in published maps and institutional affiliations. 